\title{
Simplified Hybrid Laminar Flow Control for the A320 Fin Part 2: Evaluation with the $e^{N}$-method
}

\author{
Geza Schrauf* and Heiko von Geyr ${ }^{\dagger}$ \\ DLR Institute of Aerodynamics and Flow Technology, 38108 Braunschweig, Germany
}

\begin{abstract}
A flight test with a simplified HLFC system on the vertical tail plane of an A320 aircraft was performed in April/May 2018. In this paper we discuss the preparation of the flight test data for evaluation with linear stability theory on the basis of sample measurements. Then we present our correlated transition $\mathrm{N}$-factors and compare them with the results of the A320 flight tests of 1998.
\end{abstract}

\section{Nomenclature}

$C F=$ Cross flow

$p=$ Pressure, $\mathrm{Pa}$

$w=$ Averaged suction velocity, $\mathrm{m} / \mathrm{s}$

$h=$ Flight altitude, $f t$

$M=$ Mach number

$R e=$ Reynolds number

$T=$ Temperature, ${ }^{o} \mathrm{~K}$

$T S=$ Tollmien-Schlichting

$\beta=$ Side slip angle, degree

$\delta=$ Rudder deflection angle, degree

$\mu=$ Viscosity. $\mathrm{kg} /(\mathrm{m} * \mathrm{~s})$

$\rho \quad=$ Density, $\mathrm{kg} / \mathrm{m}^{3}$

Subscripts:

$q_{c}=$ Subscript ' $\mathrm{c}$ ' denotes a quantity in a suction chamber

$q_{d}=$ Subscript 'd' denotes a quantity in the plenum or duct

$q_{s}=$ Subscript 's' denotes a quantity on the outer side of the microperforation

$q_{0}=$ Subscript ' 0 ' denotes a reference quantity

$q_{\infty}=$ Subscript ' $\infty$ ' denotes a quantity in the oncoming freestream

\section{Introduction}

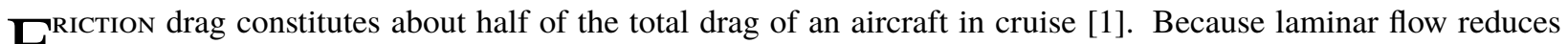
Friction drag, it has the potential to significantly reduce the fuel burn of transport aircraft, Whereas for smaller aircraft, laminarity can be achieved passively by suitable profile shaping, for larger aircraft, active boundary layer control by suction is necessary. For those aircraft, laminarity can be obtained by applying suction before the front spar in combination with a suitable shape of the wing box. The applicability of hybrid laminar flow control (HLFC) systems was shown in the 1990s by flying with such a system, for example, on the wing of a Boeing 757 [2] and, later, on the vertical tail plane (VTP) of an A320 [3, Fig. 1]. A good overview of the technology status of that time, can be found in [4]. Because those suction systems were very complex, heavy, and difficult to manufacture, simpler and lighter systems needed to be developed to make hybrid laminar flow control (HLFC) feasible for commercial use.

\footnotetext{
*Research Scientist, DLR Institute of Aerodynamics and Flow Technology, Lilienthalplatz 7, 38108 Braunschweig, Germany, AIAA Senior Member.

${ }^{\dagger}$ Head of Department, DLR Institute of Aerodynamics and Flow Technology, Lilienthalplatz 7, 38108 Braunschweig, Germany.
} 


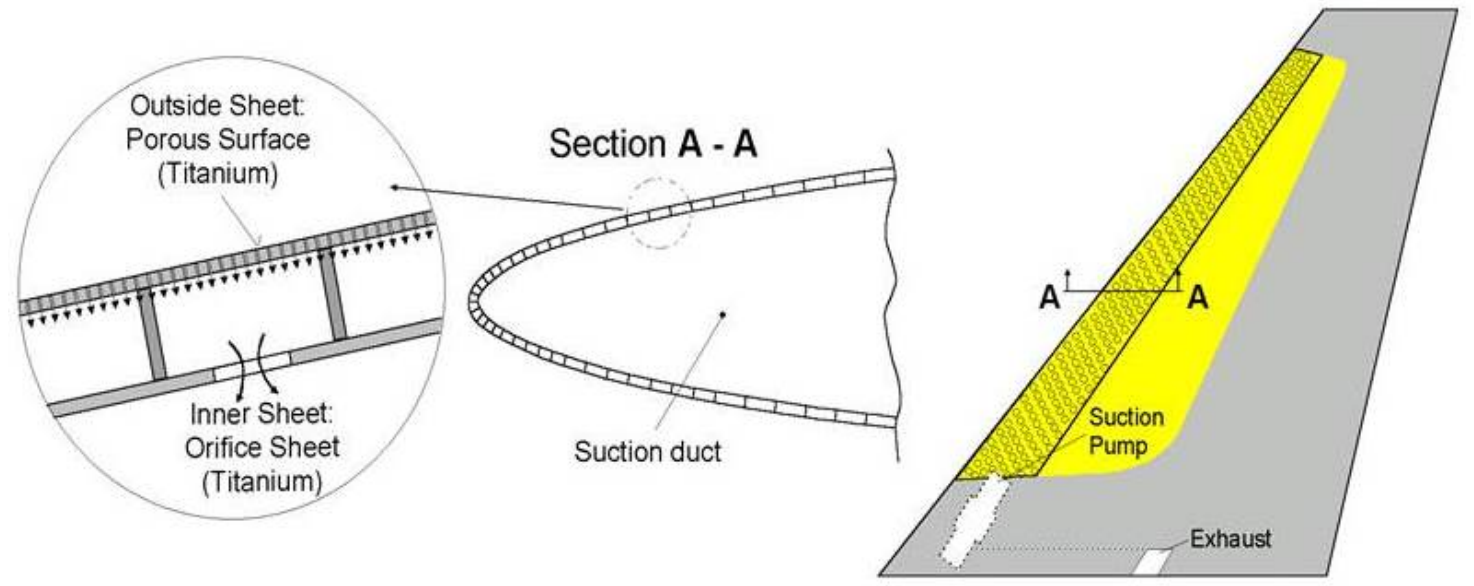

Fig. 1 Sketch of the simplified ALTTA system.

A major step towards simplification was the "simplified suction system," already proposed by Horstmann and Schröder [5] in 2001 within the European ALTTA project. A sketch of this system is shown in Fig. 1] They replaced the complex suction system used in previous flight tests by a simple double skin structure. The outer skin is, as before, a microperforated titanium sheet which is supported by an inner sheet with stringers. According to Horstmann, the small compartments created by the stringers should also act as suction chambers. This can be achieved with the help of throttle orifices. Thus, the complex system consisting of suction chambers, tubes, and valves is no longer necessary and can be replaced by an empty space: the plenum or suction duct. The challenge of this concept is that we no longer have the option of controlling the suction with the help of valves. The microperforation, the stringer locations, and the throttle holes must be balanced so that adequate suction is generated for all flight conditions. At that time, we could only show theoretical feasibility with numerical tools. Later, we were able to refine this concept as explained in Schrauf and von Geyr [3] and to build a full-scale wind-tunnel model of an A320 VTP. In 2014, we could test this model at realistic flight Reynolds numbers in the Large Low-Speed Facility (DNW-LLF) in the Netherlands.

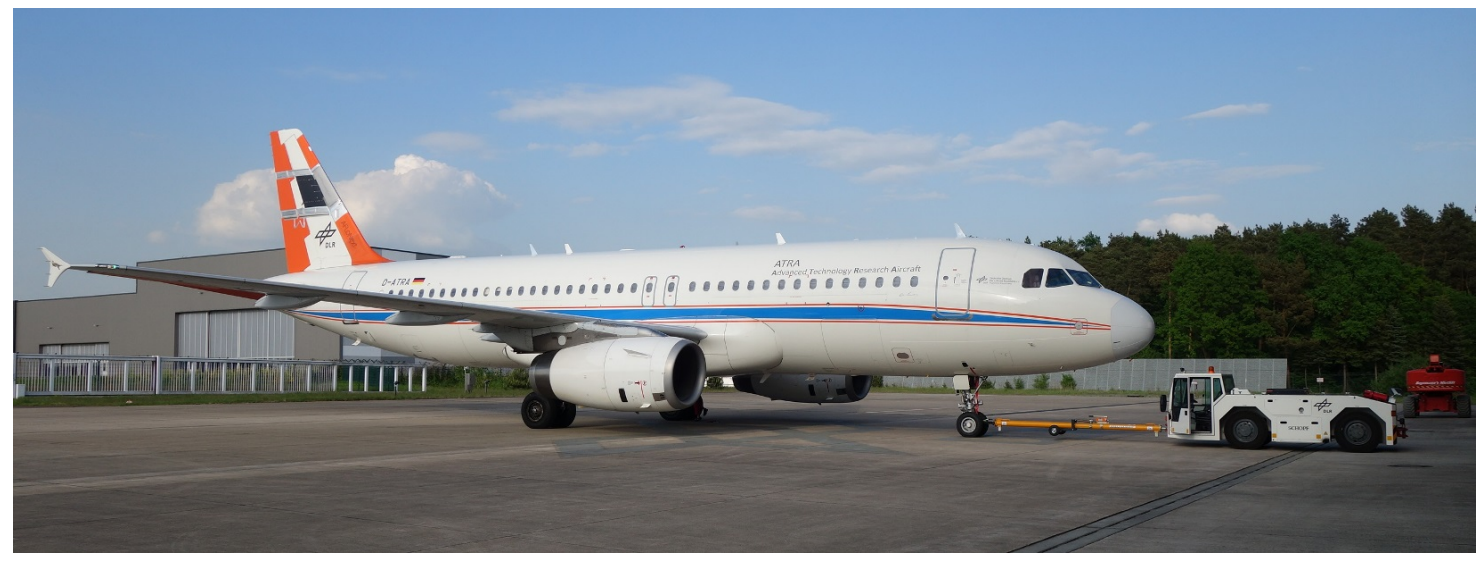

Fig. 2 DLR A320 aircraft with HLFC system installed in the VTP.

The next step was a flight test demonstration within the European AFLoNext project based on a simplified HLFC system installed in the VTP of an A320 aircraft shown in Figure 2. The aerodynamic and systems design of the HLFC leading edge was described in our previous paper [6]. Now we will report on the flights tests and their evaluation with linear stability theory. 


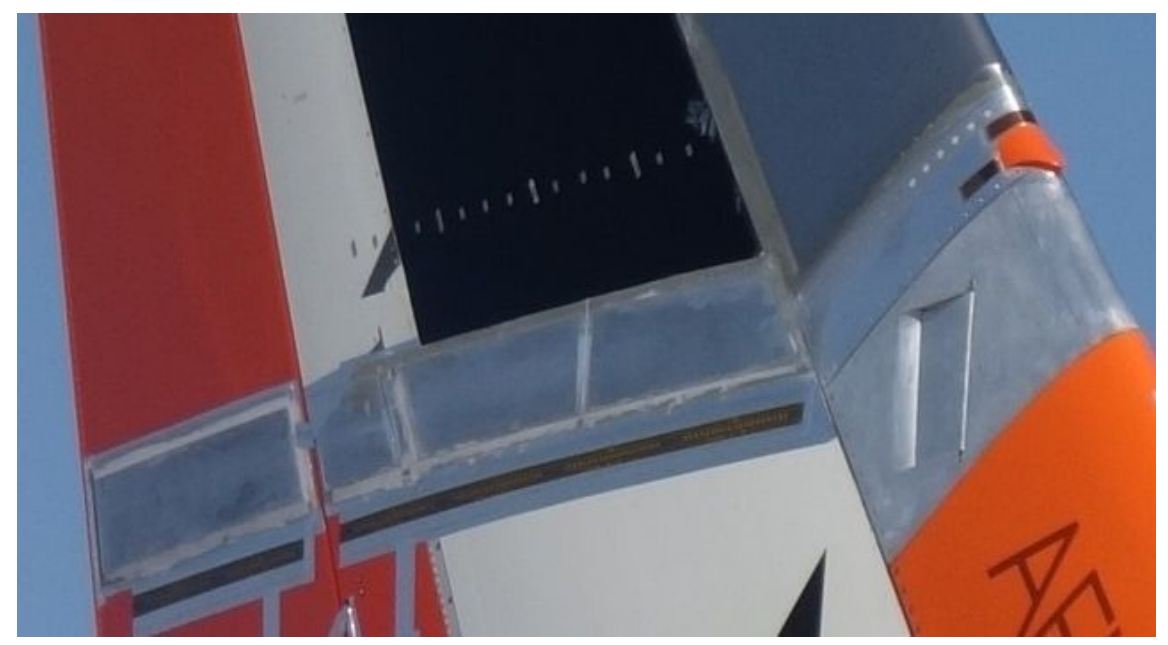

Fig. 3 Suction flap on starboard side.

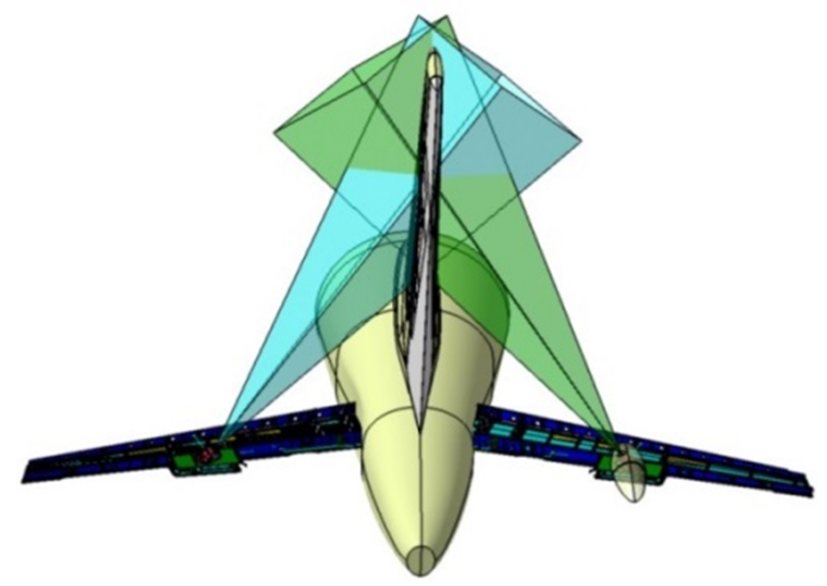

Fig. 4 Infrared cameras mounted in the horizontal tail planes.

\section{Flight Test Instrumentation}

Suction power for the HLFC system could be generated in two different ways. First, in an active mode, in which suction was generated with the help of an array of small, off-the-shelf compressors that were already certified for flight. In the second or passive mode, suction was generated with a flap on the starboard side, shown in Fig. 3. which could be opened and closed from the cabin.

The functionality of the suction system was observed with the following sensors:

- Two infrared cameras for simultanous transition detection on both sides of the VTP, installed in the horizontal tail plane (cf. Fig. 44

- Two sections with classical pressure taps: DV1 at 39\% span and DV2 at 67\% span (cf. Fig. 5p)

- In each suction chamber, two classical pressure probes as well as two MEMS sensors measuring temperature, pressure, and humidity at $25 \%$ and at $75 \%$ span

- In the plenum or duct, two classical pressure probes and also two MEMS sensors measuring temperature, pressure, and humidity at $25 \%$ and at $75 \%$ span

- A mass flow meter before the compressor, for active suction only

- Hot film sensors to check the laminarity of the flow at the attachment line

- Additionally, an experimental MEMS pressure belt on each side of the VTP box below DV1 


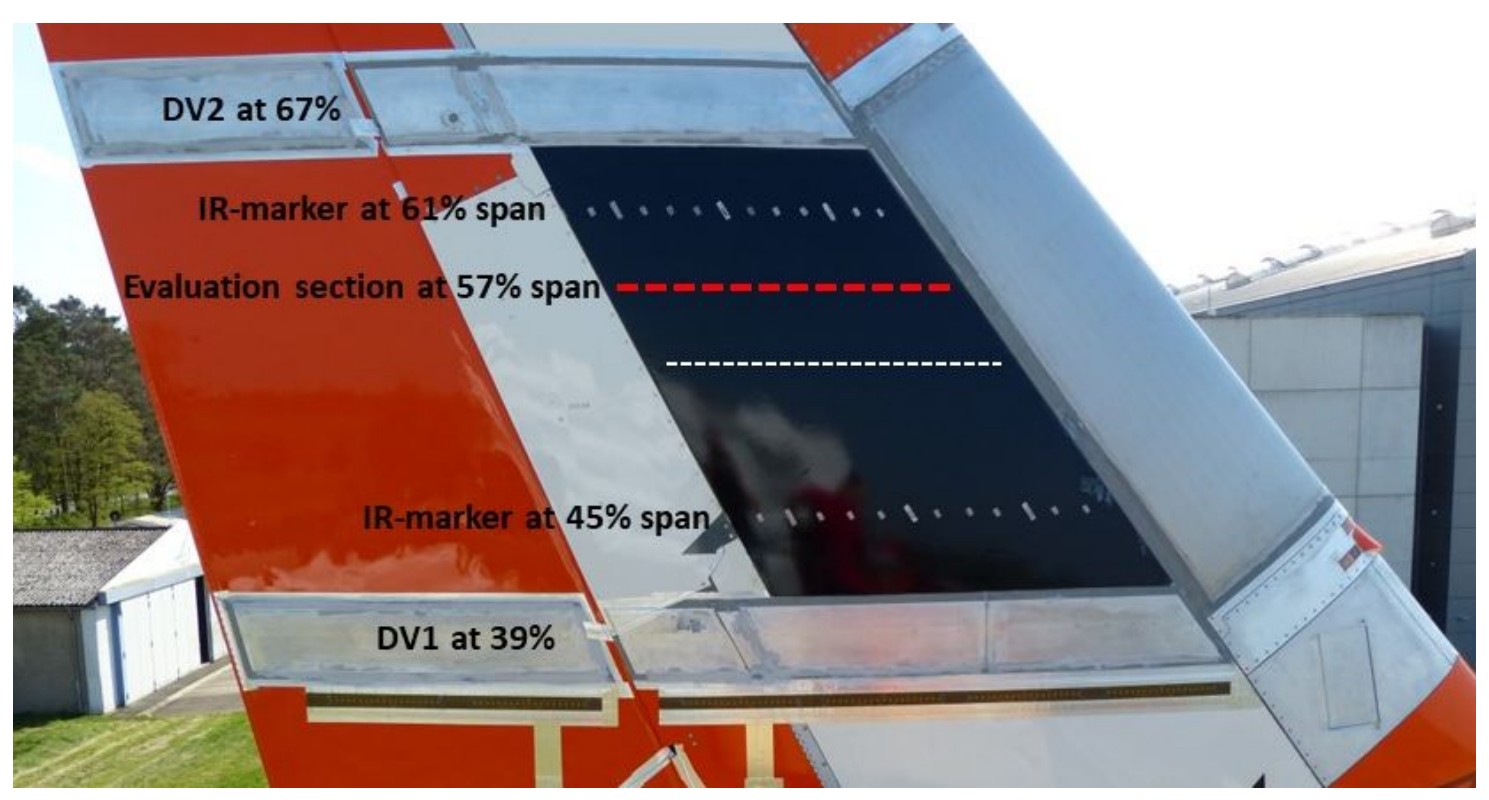

Fig. 5 Pressure sections DV1 and DV2 on starboard side.

\section{Data acquisition and processing}

\section{A. Data structure: M[measurement] files}

As in every flight experiment, we have two types of data. First, there is common data which is the same for all flight tests measurements: the geometry of the VTP, the locations of the pressure taps, the geometry of the suction chambers, especially the locations and the widths of the stringers, as well as the pressure loss characteristic of the micro-perforation on each chamber. Second, there is data specific for each flight measurement, such as
A flight altitude, Mach number, sideslip angle, free-stream pressure and temperature, ...
B external pressures, chamber pressures, ...
C chamber temperatures, chamber humidities, ...

and the infrared images. Data "A" is from the standard ATRA FTI-system (Flight Test Instrumentation system). It was continuously recorded as an ASCII FTI-data stream. Each data item can be identified by date and time. Data "B" comes as a continuous binary data stream from the pneumatic pressure system installed in the VTP, again, with date and time as identifier for each data point. A third ASCII data stream contains the data of the MEMS in the suction chambers and in the plenum.

The three data streams were recorded continuously for each flight. To ease the handling of the large amount of data, we produce for each measurement for which an infrared image is available, a small Measurement- or M-file with the associated measured data. The size of the M-file is only $6 \mathrm{kB}$. Based on the time at which the infrared images for port and starboard side were taken, we first extract the pneumatic pressures recorded at the same time ${ }^{*}$ and store these pressures together with their time stamp in an ASCII-file (cf. Fig. 6a). This is done manually on a Windows PC with the help of the program "Quicklook" [7]. In a second step, based on the pressure time, we extract the corresponding FTI data, such as flight altitude, Mach number, side slip angle, static pressure and temperature, etc., from the FTI data stream and the corresponding chamber pressures, temperatures, and humidities from the MEMS data stream. This is done on a Linux computer with a master bash script, calling several sub-scripts and a FORTRAN program (cf. Fig. 6b. The resulting M-file contains all available data (including their time stamps) for one flight test measurement linked to an infrared image. The file is written in ASCII format, so that it can be checked with any text editor. All M-files for one test flight are stored in an M-file repository.

If we find an error in the data processing, we simply assemble new M-files, which are the basic input for the automatic calculations with boundary layer and linear stability theory.

\footnotetext{
*Several pressure measurements are recorded during the time span necessary to take one infrared image.
} 


\section{Infrared Image \\ with time stamp}

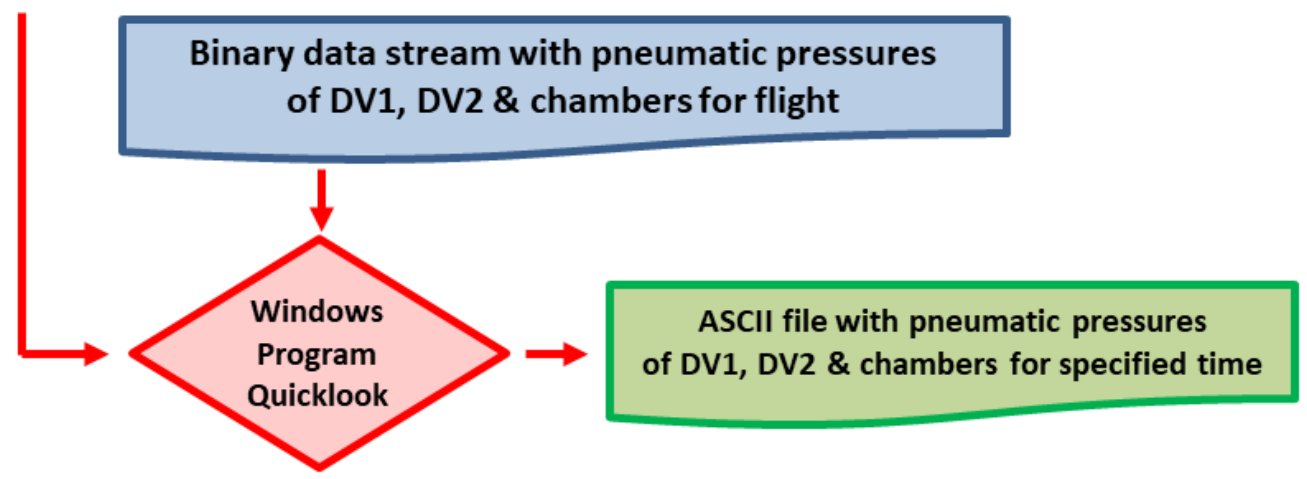

(a) Step 1.

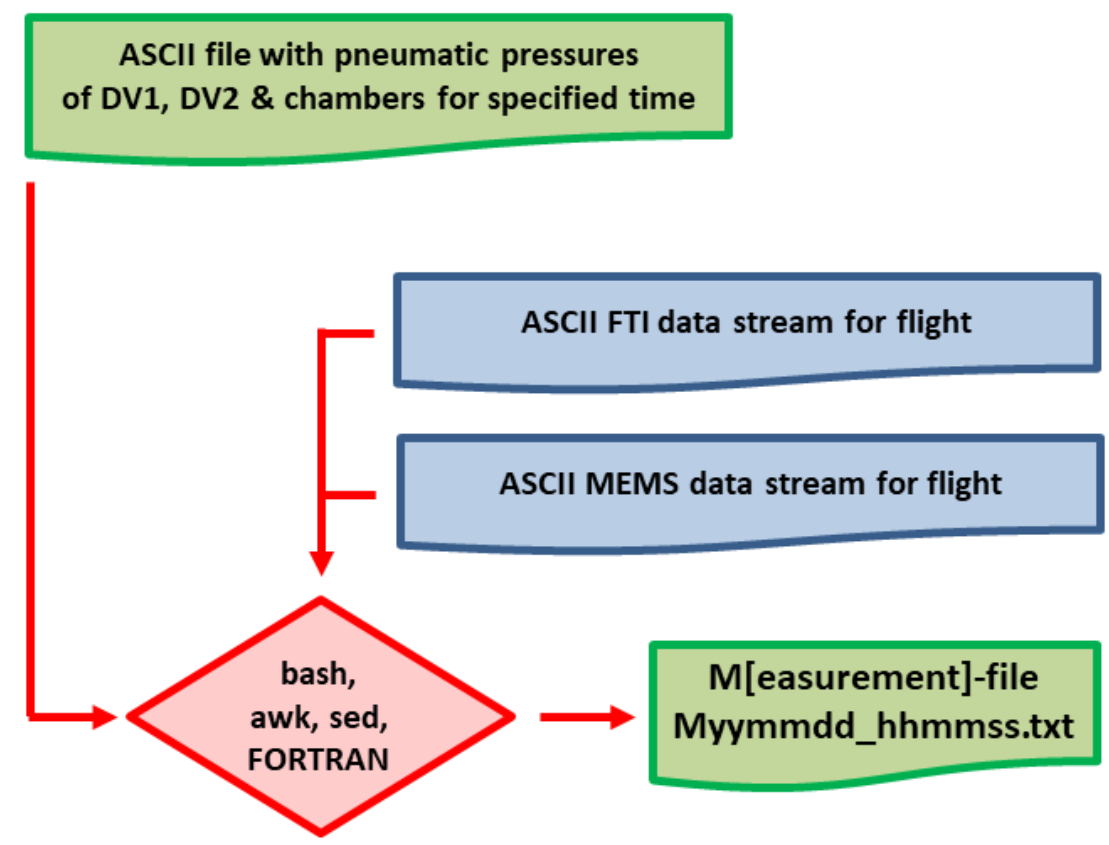

(b) Step 2.

Fig. 6 The two steps we use to assemble an M-file. 


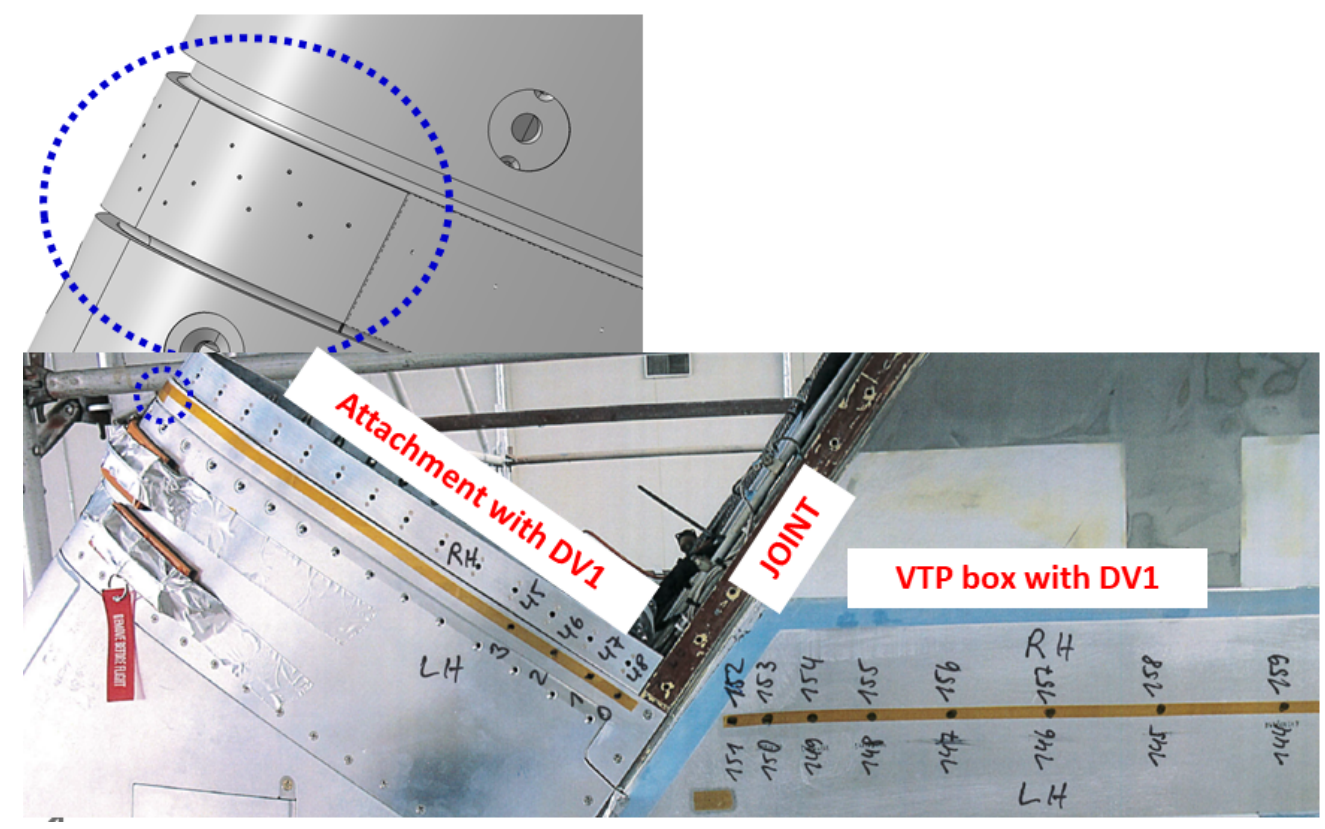

Fig. 7 Pressure taps on port side of DV1.

\section{B. Spanwise pressure distribution}

For evaluation using boundary layer and linear stability theory, we need to be able to run a boundary layer calculation for any prescribed spanwise section between the two pressure sections DV1 and DV2 (cf. Fig. 55). To do this, we need to generate an input pressure distribution for the prescribed section. This can be done with two very different methods:

Method 1: The VTP has a conical shape and the pressure distribution exhibits conical symmetry. Therefore, we can use "conical interpolation" to produce a pressure distribution for a any spanwise section.

Method 2: For each specific measurement, we could compute a 3D pressure distribution that reproduces the measured pressures at the two sections DV1 and DV2. From this 3D pressure distribution, we could then obtain a pressure distribution for any chosen spanwise section.

We use Method 1 in form of a two-step procedure based on conical interpolation, because it is much faster than Method 2 .

Behind the front spar, the pressure taps of DV1 and DV2 are already aligned along a constant-span section. However, on the metallic leading edge, the taps are aligned perpendicular to the front spar. At the leading edge, they are even staggered, as can be seen in Fig. 7 Therefore, we first apply a conical projection for the pressure taps on the leading edge, as shown in Fig. 8a and obtain two spanwise pressure distributions we call $\overline{D V 1}$ and $\overline{D V 2}$. This first step is accurate because the projection is applied for only short distances at the leading edge, where the flow complies with conical symmetry. In the second step, we perform a spanwise interpolation as shown in Fig. $8 \mathrm{~b}$. This can be done in our case, because of the conical symmetry of the flow between the two sections. However, if DV1 were located at a much lower spanwise location, the conical symmetry condition would be violated as a result of the proximity of the fuselage and the horizontal tail plane.

\section{Suction distribution}

As explained in [6], we use the pressure-loss characteristic in the form

$$
\Delta p=p_{s}-p_{c}=A \frac{\mu_{s}}{\mu_{0}} w_{s}+B \frac{\rho_{s}}{\rho_{0}} w_{s}^{2} .
$$

to model the flow through the micro-perforation. This has allowed us to separate the system design from the development of the capabilities of the manufacturer. The final values of the coefficients are $A=12400 \mathrm{~kg} /\left(\mathrm{m}^{2} \mathrm{~s}\right)$ and $B=18270 \mathrm{~kg} \mathrm{~s} / \mathrm{m}^{3}$ [6]. By inverting formula [1], we obtain the suction velocity $w_{s}$. As input for this calculation, we

\footnotetext{
${ }^{\dagger}$ The reference quantities are the values of the standard atmosphere at sea level, i.e. $\mu_{0}=1.79210^{-5} \mathrm{~kg} /(\mathrm{ms})$, and $\rho_{0}=1.225 \mathrm{~kg} / \mathrm{m}^{3}$.
} 


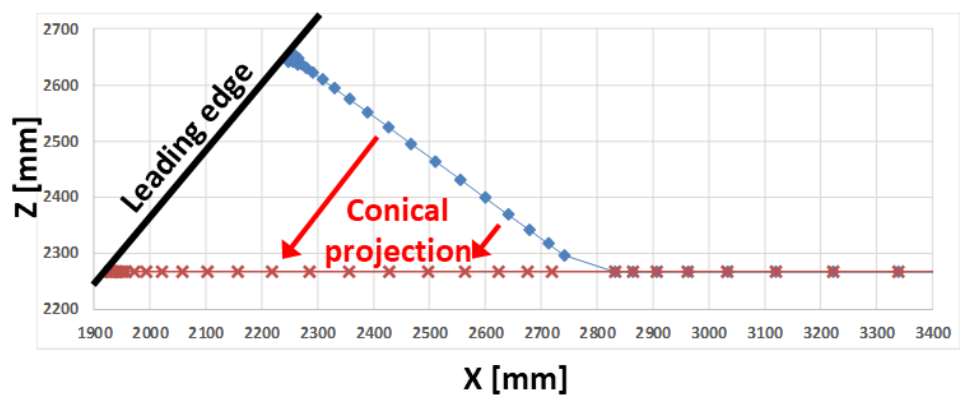

(a) Step 1: Conical projection on DV.

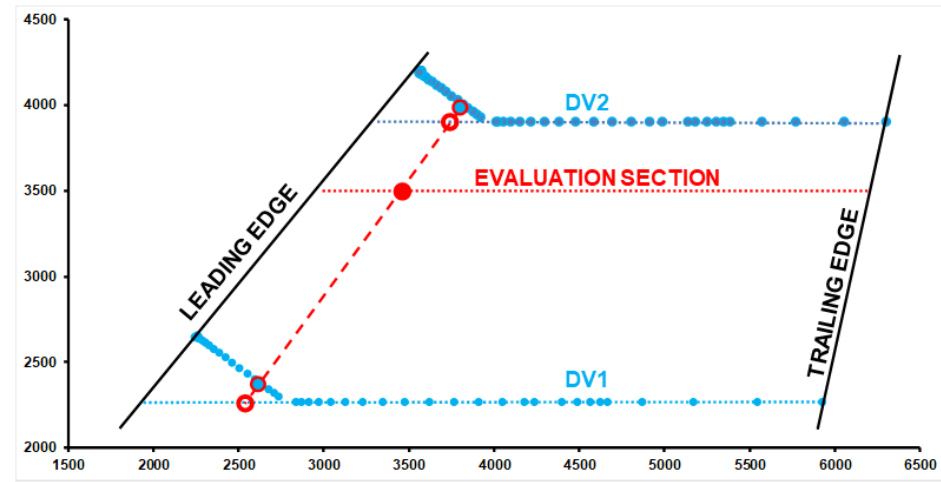

(b) Step 2: Conical interpolation.

Fig. 8 Generation of a pressure distribution at a prescribed spanwise section.

need the pressure $p_{s}$ along the selected section, the chamber pressure $p_{c}$ in the suction chamber, and the viscosity $\mu_{s}=\mu\left(T_{s}\right)$ of the fluid just above the suction surface. The temperature $T_{s}$ can be obtained by an approximation with the recovery temperature ${ }^{\circ}$. Furthermore, we have to take the stringers into account. They were laser-welded to the micro-perforation, and there is, of course, no suction where the micro-perforation is blocked by the weld lines. The computational grid for the boundary layer calculation should, therefore, be fine enough to resolve these no-suction zones $\$$

\section{Generation of input for boundary layer calculations and stability analysis}

With the methods explained in sections IV.B and IV.C we can set up a tool chain for the evaluation of the flight tests. The first step is to select one or more measurements with the help of the infrared transition visualization and to select a spanwise section at which transition can be clearly identified. For each measurement (identified by date and time), we then perform the following steps:

1) Collect the data for the measurement into an M-file.

2) Combine the pressures from the M-file with the geometry data and perform the conical projection described in Fig. 8a to obtain two CP-files for the 39\%- and 67\%-sections.

3) Perform the spanwise interpolation shown in Fig. $8 \mathrm{~b}$ to obtain a CP-file $e^{\text {Tl }}$ for the selected evaluation section.

4) Check the pressure distribution at the leading edge and compute the effective sweep angle [8, 9].

5) Compute the suction velocities using formula (1) and assemble a boundary-layer input file (BLI-file).

\footnotetext{
The recovery temperature is sufficiently accurate for the computation of $\mu\left(T_{S}\right)$ in this step.

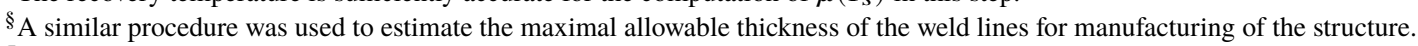

II A CP-file contains the coordinates of the points on the spanwise section and the pressures at these points.
} 
When the boundary layer input file is compiled, we can then proceed with a linear stability analysis in the usual way:

6) Perform a boundary-layer calculation with $\mathrm{COCO}[10]$.

7) Perform a stability analysis with LILO [11].

8) Correlate the transition $N$-factors with the help of the transition location of the infrared image.

With this algorithmic set-up we are now able to perform the boundary layer and the stability calculations necessary for the evaluation. This is, however, only the numerical prerequisite. In order to obtain correct results, we have to carefully analyze the recorded values of each measurement, as will be shown in the next section.

\section{E. "Cleaning" of the pressure distribution}

\section{Locations of pressure taps}

Boundary layer stability is very sensitive to local flow acceleration and deceleration, dependent on the local pressure gradient. Therefore, the measured pressure distributions must be carefully analyzed in order to make corrections where needed.

Determination of the correct coordinates of the pressure taps is a difficulty we encountered in all flight experiments within the last decades, These coordinates are important, because the local pressure gradient depends not only on the measured pressure values but also on the distance between neighboring taps. It is a standard procedure to set up a table mapping the number of each pressure tap with the number of the corresponding channel of the pneumatic pressure system and also with the dimensional coordinates of the pressure tap from the CAD-system. From the coordinates, we can compute the distances between neighboring taps. These were checked as follows: after the installation of the HLFC system on the aircraft, we used a cherry picker to attach transparent foils on the sides of the VTP covering the taps. Then we marked the taps with a pen. After removing the foil, we measured the distances between the marked points on the foil and compared them with the distances obtained from the CAD-data. We observed inconsistencies and had to re-work the coordinates several times. Most discrepancies had the following two causes:

1) The pressure taps on the VTP box were installed in a "pressure belt" and these belts were attached with a slight offset compared to the CAD-data.

2) We requested as many taps at the leading edge, as can be seen in Fig.77. Due to space allocation problems, the taps were drilled at slightly different locations than originally planned.

The effort to determine the correct locations of the pressure taps should not be underestimated. In our case it took several rounds. The correct locations were finally confirmed after the first flight.

\section{Disregarded pressure taps}

It is self-evident that we have to omit faulty pressure taps from the evaluation. There are, however, additional challenges. In Fig. 9 we present a close-up of the starboard side with the beginning of the pressure belt and the suction flap. The pressure belt had a thickness of $3 \mathrm{~mm}$ and, thus, acted as forward facing step. This step can be seen in the measured pressure distribution: the first pressure tap on the belt was at $22.5 \%$ chord where the pressure distribution exhibits a characteristic peak as can be seen in Figs. 10a and 10b The effect is still visible in the following three pressure taps. Altogether, the four taps, marked with black circles, are affected before the step-effect dies out.

The suction flap caused a disturbance of the pressure distribution which was much larger than expected. Due to space limitations, the flap was installed close to the pressure distribution DV1, just below the HLFC leading edge (cf. Fig. 5). The flap closed on an inward ramp, i.e. it was not flush mounted. Therefore, it influenced the pressure distribution even when closed, i.e. when the HLFC system was running in active mode. In passive mode, the influence became more noticeable with larger opening angles. In Figs. 10a and 10b we present a sequence of measurements taken within only ten minutes. The aircraft was flying in stabilized condition with Mach number 0.78 at a flight altitude of $39000 \mathrm{ft}$. We clearly see that the influence of the flap on the pressure distribution is present up to $5 \%$ chord. It affected eleven pressure taps (marked with red circles).

The area on which transition was visualized is the black foil between the two pressure sections DV1 and DV2 (cf. Fig. 57. In this area the surface was smooth and free of any disturbancell]

\footnotetext{
" During the flights we observed some issues at the joint between the leading edge and the VTP box. The order of magnitude was in micrometer, not millimeter or centimeter. Hence, they probably only had a very minor effect on the pressure distribution.
} 


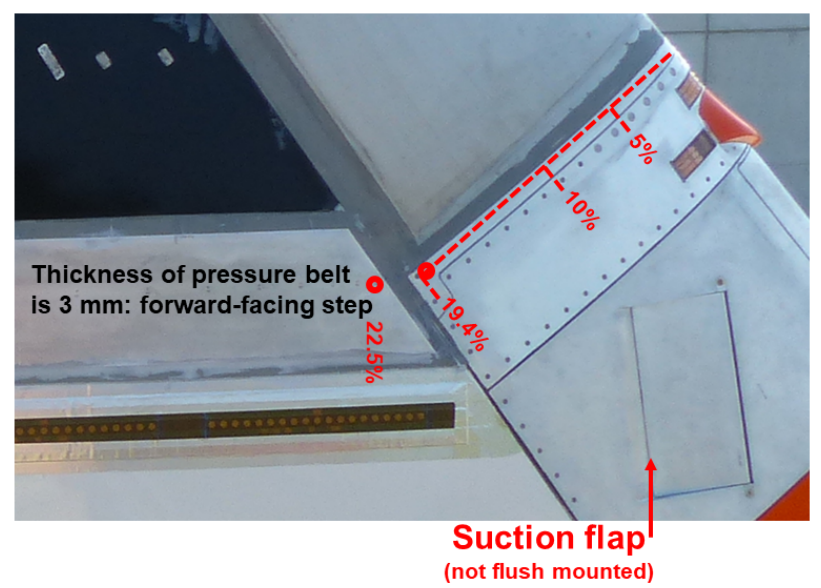

Fig. 9 Close-up of pressure belt and suction flap.

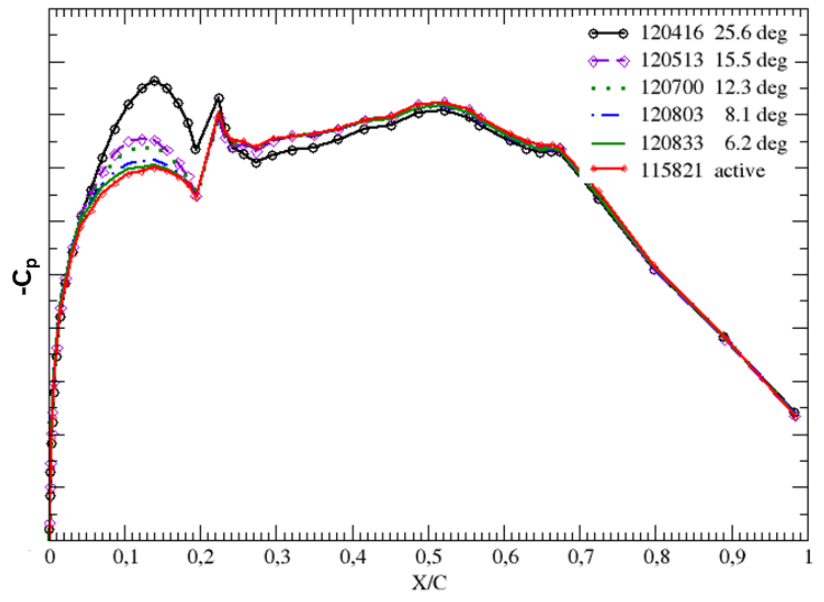

(a) $C_{p}$-distribution for several flap angles.

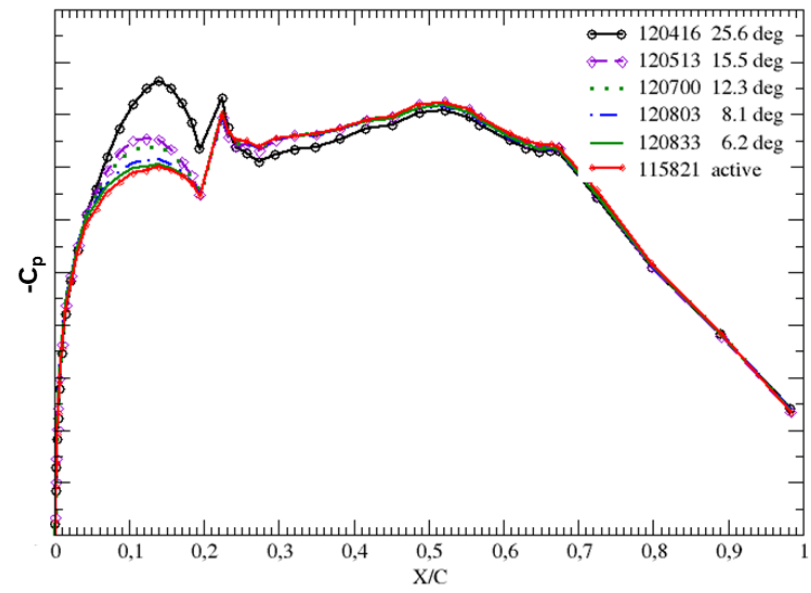

(b) Details of above

Fig. 10 DV1 pressure distributions for flight 549, $39000 \mathrm{ft}$, Mach 0.78 on starboard side. 


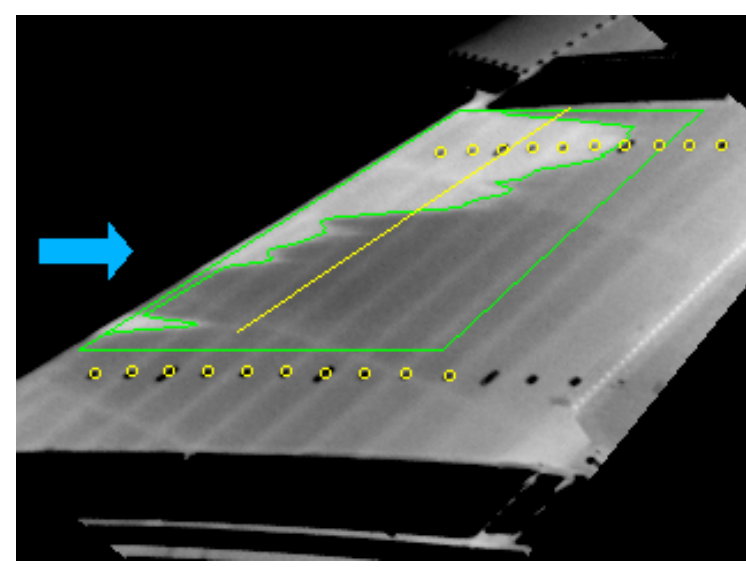

Fig. 11 Infrared image of port side.

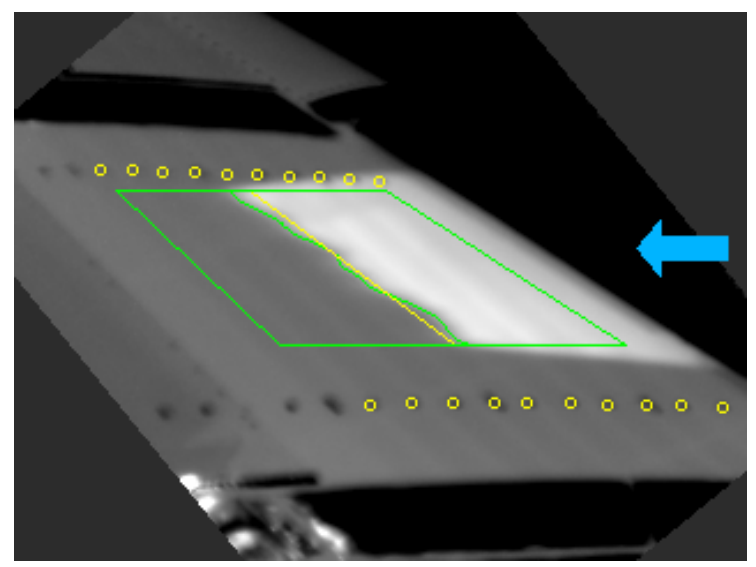

Fig. 12 Infrared image of starboard side.

$$
h=31000 \mathrm{ft}, \quad M=0.78, \beta=0^{o} \text {, strong active suction. }
$$

In view of these effects, we excluded the four black taps and also the eleven red pressure taps from the evaluation and bridged the gap with an Akima interpolation [12]. This process is justified in our case because we apply boundary layer suction before the front spar. The suction damps the cross-flow amplification so that the transition observed on the VTP-box is caused by the Tollmien-Schlichting (TS) instability. Its neutral point occurs downstream of the suction panel. Furthermore, we see from Fig. 10a, that the gradient of the pressure distribution on the VTP box, is hardly affected. Therefore, we find only minor influence on the Tollmien-Schlichting $\mathrm{N}$-factors caused by the modification of the pressure distribution,

Each forward edge of the other three pressure belts also created a forward facing step causing a footprint in the pressure distribution. Thus we had to disregard a few taps. However, for those edges, the effect is much weaker:

\section{Spanwise variation of the transition line}

In Figs. 11\& 12, we present the infrared transition visualization for a symmetric case, i.e. with side slip angle $\beta$ and rudder deflection angle $\delta$ approximately $0^{\circ}$. We were flying at $31000 \mathrm{ft}$ with Mach number 0.78 . During this measurement, strong active suction was applied. On the starboard side, transition occurs, as expected, over the whole span along a constant-chord line at around 38\% to $40 \%$. The port side shows a different picture: on the upper part of the visualization area, transition occurs at $39 \%$, i.e. at the same chordwise location as on the starboard side. However, farther down, transition moves forward and occurs before or at the joint of the HLFC leading edge and the VTP-box. This early transition is probably caused by some surface imperfection. Regarding the evaluation of such a case, we pose the two questions:

Q1 Can the variation of the transition line on the port side be explained with linear stability theory?

Q2 How do we correlate a transition $\mathrm{N}$-factor for such a case?

To answer these questions, we perform an analysis with boundary layer and stability calculations at different spanwise sections. We choose three sections at 53\%, 57\%, and 61\% span, where transition occurs at $25 \%, 35 \%$, and $39 \%$. In Fig. 13. we present the transition line obtained with our photo-metric method together with the three evaluation sections, and in Fig. 14, a projection on the $(x, z)$-plane to rectify the image. The calculated Tollmien-Schlichting $N_{T S}$-factors are shown in Fig. 15. We see that the pressure distributions (blue lines) obtained for the three sections are quite similar. Therefore, it is no surprise that we get very similar $N_{T S}$-factor秚envelopes, i.e. the forward movement of the transition is not reflected in the $N$-factor results. A straightforward correlation of the $N$-factors would give the following results:

S61 at 61\% span: transition at 39\% $\Longrightarrow N_{T S}=9.0$.

S57 at 57\% span: transition at 35\% $\Longrightarrow N_{T S}=7.7$.

S53 at 53\% span: transition at $25 \% \Longrightarrow N_{T S}=3.6$.

** We only show the $N_{T S}$-factors, because, due to the suction, the $N_{C F}$-factors are so small that transition is induced by the Tollmien-Schlichting instability (see, for example, Figs. 23c \& 23d. 


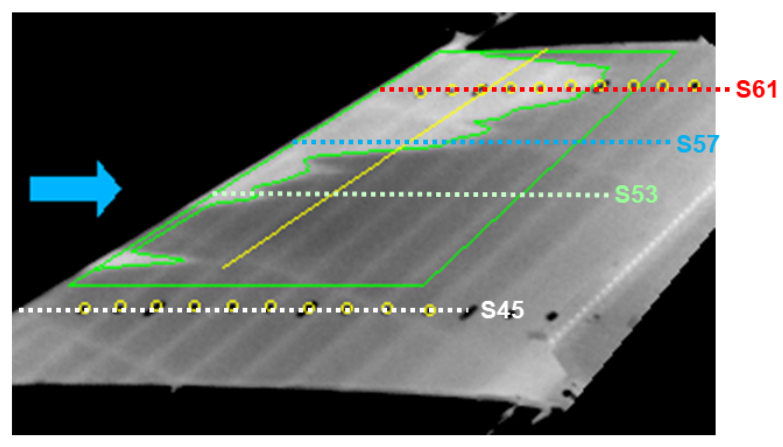

Fig. 13 Selected evaluation sections.

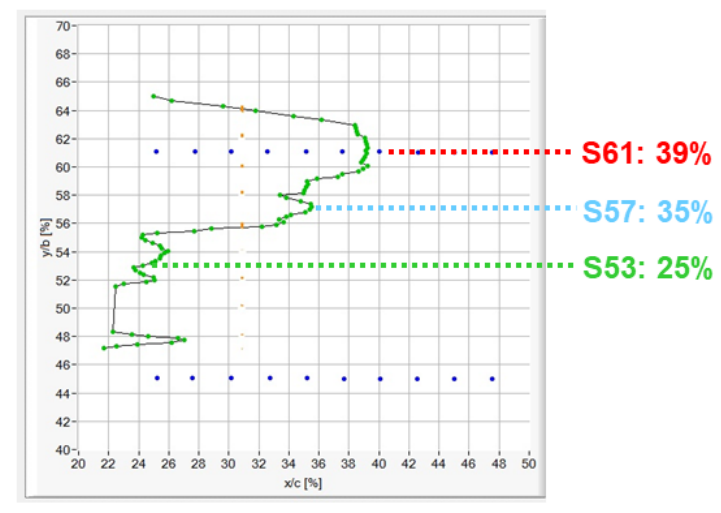

Fig. 14 Transition line and evaluation sections.
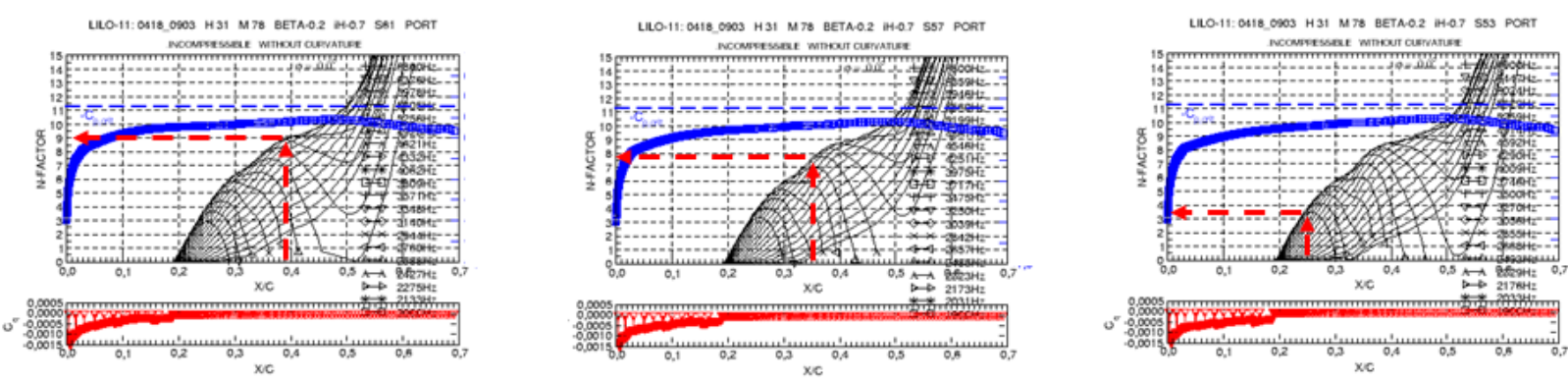

Fig. 15 Pressure distributions and $N_{T S}$ factors for the three evaluation sections.

We see that the correlated transition $N$-factors are very different, even though the stability situation in the three sections is similar. In order to explain the different transition locations with linear stability theory, we would expect that the forward shift of transition is reflected in somewhat larger $N$-factors at $57 \%$ and much larger ones at $53 \%$. This is obviously not the case, i.e. the variation of the transition line is not reflected in the stability results. Because the numerical input for the calculations is similar, the numerical output is also similar. This means that the imperfections causing the shift of the transition line ${ }^{+1}$ are not modeled by our procedure consisting of boundary layer and stability calculations.

Therefore, we propose to use only section S61 for the $N$-factor correlation. This means that with linear stability theory we obtain the "laminar potential" of the boundary layer flow, which can be reached without imperfections. By choosing the "best section" for the correlation, we differ from earlier evaluations, in which we determined one so-called "evaluation section" for all measurements. Maybe this is one reason for the large scatter in the correlated $N_{T S}$-factors obtained in earlier results.

\section{Surface temperature}

Infrared transition visualization was already used for the A320 flight tests in 1998 with the research HLFC system [13]. However at that time, we installed heater mats on both sides of the VTP-box. The mats marginally increased the surface temperature. Because heat transfer in a turbulent boundary layer is larger than that in a laminar one, areas with turbulence will be cooled a little more. Thus they have a somewhat lower surface temperature than laminar areas. The small temperature difference between laminar and turbulent areas is made visible with the infrared camera.

Initially, it was planned to also use heater mats for this flight test. However, because today's infrared cameras can resolve much smaller temperature differences, we decided to use a black heat-isolating foil instead. Previous tests showed that the sun's heat would sufficiently increase the surface temperature of the black foil to apply infrared

\footnotetext{
$\dagger$ On the ground, we checked the joint with a perthometer and found that its lower part was smoother than its upper part, which is is not consistent with Figure 13 Of course, the condition of the joint in flight might be different from its condition on ground.
} 


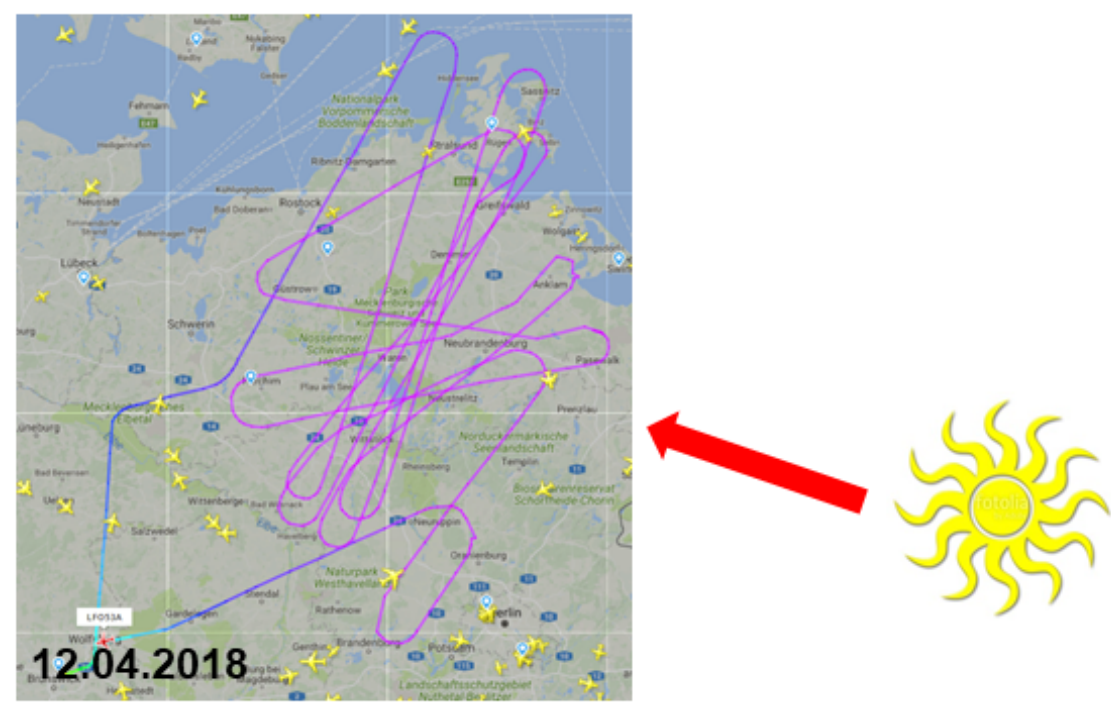

Fig. 16 Flight path with large legs perpendicular to the sun.

technique, even in northern Germany. Because we relied on sun heating, we mostly tested from late morning to early afternoon. Within our designated airspace, we flew with the sun coming from one side, as shown in Fig. 16 We found that the infrared visualization even worked for both sides, the sunny side as well as the shady side. However, the sunny side did produce a better the contrast in the infrared images.

It is known that surface heating and cooling influences the Tollmien-Schlichting transition: surface heating destabilizes the boundary layer so that TS-transition is moved forward, and cooling, on the other hand, stabilizes the boundary layer and moves TS-transition downstream. When a heater mat is used, as in the earlier flight tests, sun heating probably has only a minor influence on the surface temperature. Both sides are somewhat warmer compared to an adiabatic surface, which might result in a small forward shift of transition. In our previous evaluations [14], we did not take surface heating into account. This might have caused a slight shift of the correlated $N_{T S}$-factors towards smaller values, however, the shift would have the same magnitude for both sides. In the current flight tests without a heater mat, the difference in surface temperature between the sunny and shady side might be noticeable.

As mentioned in section [II], we evaluated the feasibility of MEMS-belts for future pressure measurements in flight testing. To do this, we attached four MEMS belts on both sides of the box and the rudder as can be seen in Fig. 5 . The positions of the sensors were not recorded because we did not intend to use their data. However, we were able to estimate the sensor positions by comparing their pressure values with the classical pressures of the neighboring section DV1. Because the MEMS sensors record both, pressure and temperature, we can get some information on the surface temperature. An example is shown in Fig.17.

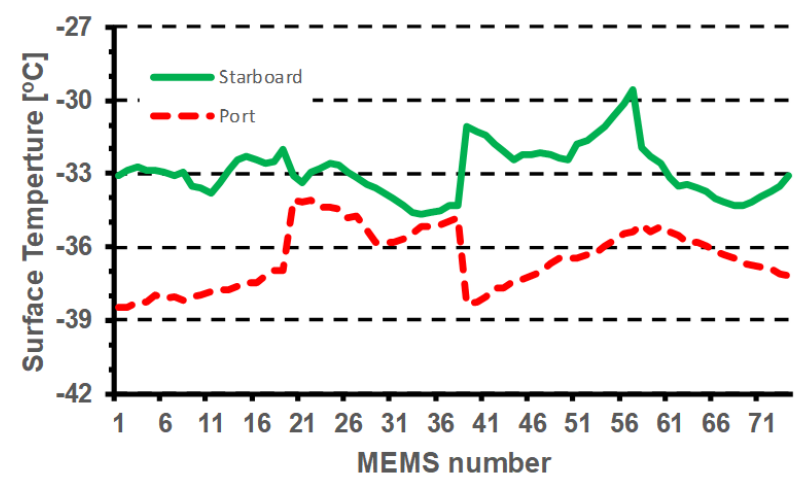

Fig. 17 Surface temperatures recorded by the MEMS belts. 
Unfortunately, the temperature sensors were not adequately calibrated, so that the temperatures exhibit non-physical jumps. Nevertheless, Fig. 17 shows a temperature difference between the two sides: according to this data, the average surface temperature on the starboard side is $-32.9^{\circ} \mathrm{C}$ and on the port side $-36.5^{\circ} \mathrm{C}$, indicating a temperature difference of $3.6^{\circ} \mathrm{C}$.

To see whether such different temperatures have an influence on the correlated $N_{T S}$-factors, we evaluate nine measurements of the same flight, flying at $35000 \mathrm{ft}$ with Mach 0.78 and sideslip angle $\beta=0^{\circ}$, using our standard procedure consisting of an adiabatic, compressible boundary layer computation followed by linear stability calculations with local, incompressible stability theory. For each measurement we correlate for port and starboard sides the $N_{T S}$-factors for the beginning and the end of transition with the help of the infrared images, and compute their mean values. Next, we calculate for each class, i.e. "starboard-sunny" (4 cases), "starboard-shady" (5 cases), "port-sunny" (5 cases), and "port-shady" (no case with sufficiently good infrared image) the average of the mean $N_{T S}$-factors and present them in Table 1. For the starboard side we obtain $N_{T S \text {, shady }}-N_{T S \text {, sunny }}=1.2$, which is in line with our assumption

Table 1 Correlated $N_{T S}$-factors averaged over ten measurements.

\begin{tabular}{|c|c|c|}
\hline Side & Sun & $N_{T S}$ \\
\hline starboard & sunny & 8.9 \\
starboard & shady & 10.1 \\
\hline port & sunny & 8.1 \\
port & shady & \\
\hline
\end{tabular}

that surface heating moves the transition forward, so that the correlated $N_{T S}$-factor becomes smaller. For this side we find an $N_{T S}$-factor shift in the order of $\Delta N=1$. Unfortunately, we have currently no values for the port side when it is shady. This might change when we evaluate more flights.

\section{Suction variation}

In this section we consider the same flight as in the two previous chapters and present the results of a suction variation. The aircraft flew with Mach number 0.78 at flight altitude $39000 \mathrm{ft}$ and with side slip angle $\beta$ close to zero. Having stabilized the flight, we opened the suction flap to $26.6^{\circ}$ and then closed it step by step until we observed a loss of transition. For each flap angle we took a measurement. All measurements were taken on the starboard side within less than five minutes. Some data is shown in the following table:

Table 2 Suction variation, transition location, and correlated $N_{T S}$-factors.

\begin{tabular}{|c|c|c|c|c|c|c|c|c|c|}
\hline No. & Time & $\delta[\mathrm{deg}]$ & $\Delta p[\mathrm{~Pa}]$ & $X_{t r, 1}$ & $X_{t r, 2}$ & $X_{\text {tr, mean }}$ & $N_{T S, 1}$ & $N_{T S, 2}$ & $N_{T S, \text { mean }}$ \\
\hline 1 & 120513 & 15.5 & 4590 & $48 \%$ & $48 \%$ & $48 \%$ & 9.4 & 9.5 & 9.45 \\
2 & 120621 & 13.2 & 3958 & $47 \%$ & $49 \%$ & $48 \%$ & 9.0 & 9.7 & 9.35 \\
3 & 120700 & 12.2 & 3741 & $47 \%$ & $50 \%$ & $48.5 \%$ & 9.3 & 10.0 & 9.65 \\
4 & 120730 & 10.3 & 3206 & $50 \%$ & $51 \%$ & $50.5 \%$ & 9.9 & 10.6 & 10.25 \\
5 & 120803 & 8.1 & 2750 & $49 \%$ & $50 \%$ & $49.5 \%$ & 9.8 & 10.4 & 10.10 \\
6 & 120833 & 6.2 & 2223 & $49 \%$ & $51 \%$ & $50 \%$ & 10.1 & 10.8 & 10.45 \\
7 & 120925 & 5.5 & 1734 & & & & & & \\
\hline
\end{tabular}

Herein, the measurement time is given in the format hhmmss. The quantity $\Delta p$, representing the suction strength, is the difference between the freestream pressure and the duct pressure, i.e. $\Delta p=p_{\infty}-p_{\text {duct }}$. We consider $\Delta p>5800 \mathrm{~Pa}$ as "very strong suction", $\Delta p>4500 \mathrm{~Pa}$ as "strong suction", and $\Delta p>3800 \mathrm{~Pa}$ as "weak suction". Depending on the sideslip angle, outflow can occur if $\Delta p<2500 \mathrm{~Pa}$. Because, even for good infrared images, the transition line exhibits some spanwise variation, we choose a suitable spanwise region and consider the variation of the transition line in this region. $X_{t r, 1}$ in column five is the earliest transition location and $X_{t r, 2}$ in column six the latest. In columns eight and nine we present the corresponding correlated $N_{T S}$-factors. Additionally, we list in column seven the mean value $X_{t r, \text { mean }}=\left(X_{t r, 1}+X_{t r, 2}\right) / 2$ and in the last column the mean value $N_{T S, \text { mean }}=\left(N_{T S, 1}+N_{T S, 2}\right) / 2$.

\footnotetext{
$\$$ We encountered the difficulty that there are slight differences in the pressure distributions between the two sides. We have to be careful not to consider measurements in which the temperature effect might be overruled by a pressure effect. An aircraft is not mathematically symmetric and always rolls a little bit. The sideslip angle for the nine cases varied between $-0.2^{\circ}$ and $+0.15^{\circ}$.
} 


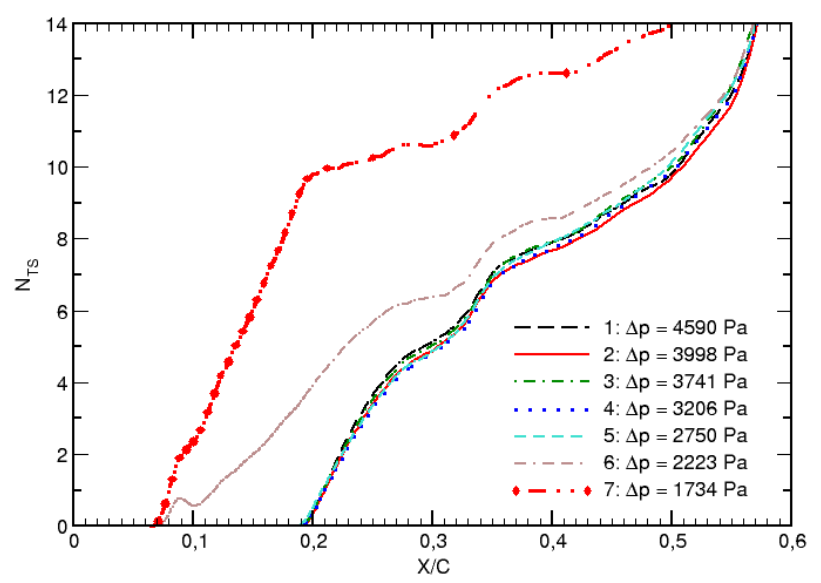

(a) $N_{T S}$-factor envelopes.

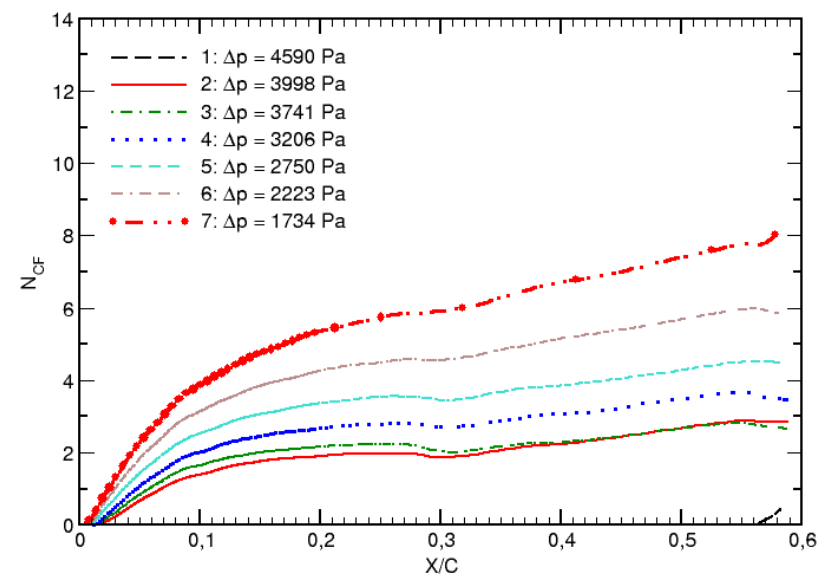

(b) $N_{C F}$-factor envelopes.

Fig. $18 N$-factor envelopes of the cases from Table 2.

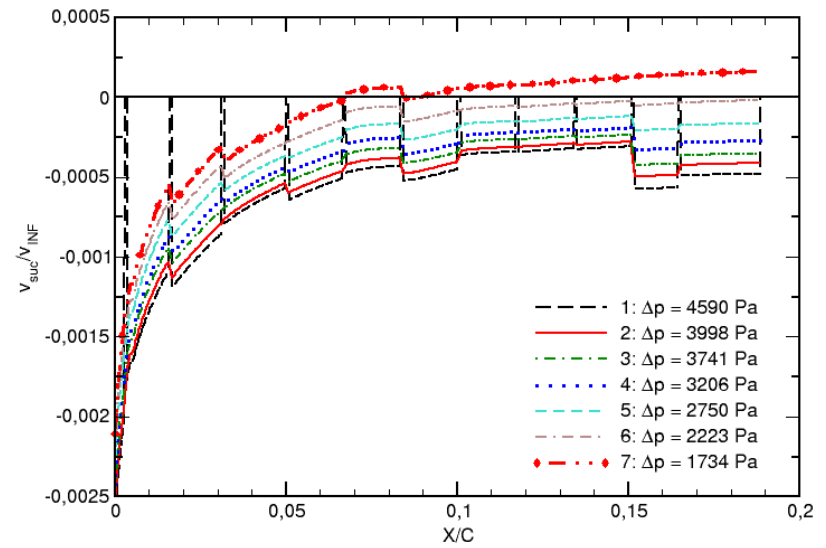

Fig. 19 Nondimensional suction velocities of the cases from Table 2. 
To easier compare the cases, we do not present the individual $N$-factor curve for each frequency as in Figure 15 , but only the envelope for each case. The envelopes of the $N_{T S}$-factor curves are shown in Figure 18a and those for the stationary crossflow modes, in Figure $18 \mathrm{~b}$. We see that, for the first five cases, the $N_{T S}$-envelopes are nearly identical. This is consistent with the observed transition locations which do not change much. The reduction of the suction strength is only seen in an increased growth of crossflow modes. However, these modes are not strong enough to influence transition. If we further reduce suction, as in case six, we observe some Tollmien-Schlichting growth on the suction panel. This does not yet influence transition.

In Figure 19 we present the non-dimensional suction velocities $w / v_{\infty}$ for those cases. We see short no-suction zones where the stringers are welded to the micro-perforated suction sheet. In order not to overload the graphic, we present only the no-suction zones for the first case, to be able identify the suction chambers. Of course, the short no-suction zones also exist for the other cases. They are, in fact, input for the boundary layer calculation $\$ \$$ For case six, suction becomes very weak in the sixth chamber $(0.068<\approx X / C<\approx 0.083)$. There is, however, no outflow. The very weak suction allows for TS-growth over the suction panel and also for larger $N_{C F}$-factors. From the infrared image we see that TS-transition still occurs at around 50\%, corresponding to a correlated $N_{T S}$-factor of 10.5 . This shows that, even for $N_{C F} \approx 5$ (obtained with incompressible stability theory), cross-flow modes do not yet influence the transition process, i.e. that linear stability theory is still valid. Stronger CF-modes can cause non-linear effects, such as an interaction $\sqrt{\text { ITII }}$ of cross-flow modes with Tollmien-Schliching waves, or a deformation of the velocity profiles of the boundary-layer flow.

The situation changes if the suction is further reduced as we see from case seven. Now we encounter global outflow over chamber 6, local outflow ${ }^{\text {采 }}$ over chamber seven, and again global outflow up to the end of the suction panel. The outflow causes a rapid growth of the $N_{T S}$-factors, and also further growth of the crossflow modes. The infrared image shows that transition occurs before the end of the metallic suction panel, and, therefore, its location cannot be determined. However, the forward shift of transition is consistent with the large $N_{T S}$-factors of the order of 10 at the end of the panel.

\section{VIII. $N$-factor correlation}

To compare transition results from different tests, we can display pairs of correlated $N_{C F}$ and $N_{T S}$-factors as shown in Figure 20, which is a reproduction of Figure 15 from [14]. The blue entries are the results from the flight tests with the natural-laminar-flow (NLF) glove on the the ATTAS/VFW614 aircraft (1989), and the black entries are from the NLF flight tests with a Fokker 100 aircraft (1996). Based on those NLF tests, the first author established the outer correlation curve (dot-dash-dash) for the design of natural laminar flow surfaces (see e.g. [15]). Because the curve is placed on the inside of the correlated $\left(N_{C F}, N_{T S}\right)$-points, it results in a conservative transition prediction, i.e. transition will most likely occur farther downstream than predicted with this curve.

The green entries represent the results of the A320 HLFC VTP flight tests from 1998 [13]. The values of the correlated $N_{T S}$-factors are relatively small. The cause remains unclear. However, it was speculated that it might have been caused by interior sound emanating through the micro-perforated suction surface into the boundary layer. The general opinion was that Tollmien-Schlichting $N$-factors in the order of 6 to 7 are to pessimistic for HLFC design. Therefore, K.H. Horstmann and the first author proposed the inner, green correlation curve (dot-dot-dash), allowing for a Tollmien-Schlichting transition $N_{T S}$-factors of 9.5. Because suction through a micro-perforated panel causes an additional roughness, we also reduced the values for the allowable $N_{C F}$-factors, even though no experimental evidence from flight tests was available. It must be emphasized that this curve was the result of an educated guess and is not based on any flight test results. Nevertheless, it has been used for HLFC-design, as for example the design pf the DNW-LLF test (cf. Figure 2 in [6], for the design of this HLFC system, and for current projects.

Our results from the present flight tests are shown in Figure 21. We see that the $N_{T S}$-factors are indeed larger than those from the 1998-test. The educated guess does not seem too bad. However, we have to evaluate the other flights before we can establish a new correlation.

There are two cases outside of the NLF-curve: The case "111915-port" has the largest $N_{T S}$-factor, larger than twelve. However, this case is somewhat unusual. Suction is very weak, the infrared image is unclear and might show the beginning of the loss of laminarity. The infrared image of the starboard side is much clearer, even though it was the shady side, which normally exhibits a weaker contrast. The other case "120833" is also one with weak suction. The transition line, determined by our photometric method, might be somewhat father downstream than a human observer would find.

\footnotetext{
$\S \S$ With similar calculations we determined the maximal allowable thickness for the weld line.

IIII Examples of non-linear interactions of Tollmien-Schlichting waves with crossflow modes can be found in [24].

***We distinguish between local and global outflow. With local outflow, there is still suction over some parts of the chamber, whereas global outlow means outflow over the whole chamber.
} 


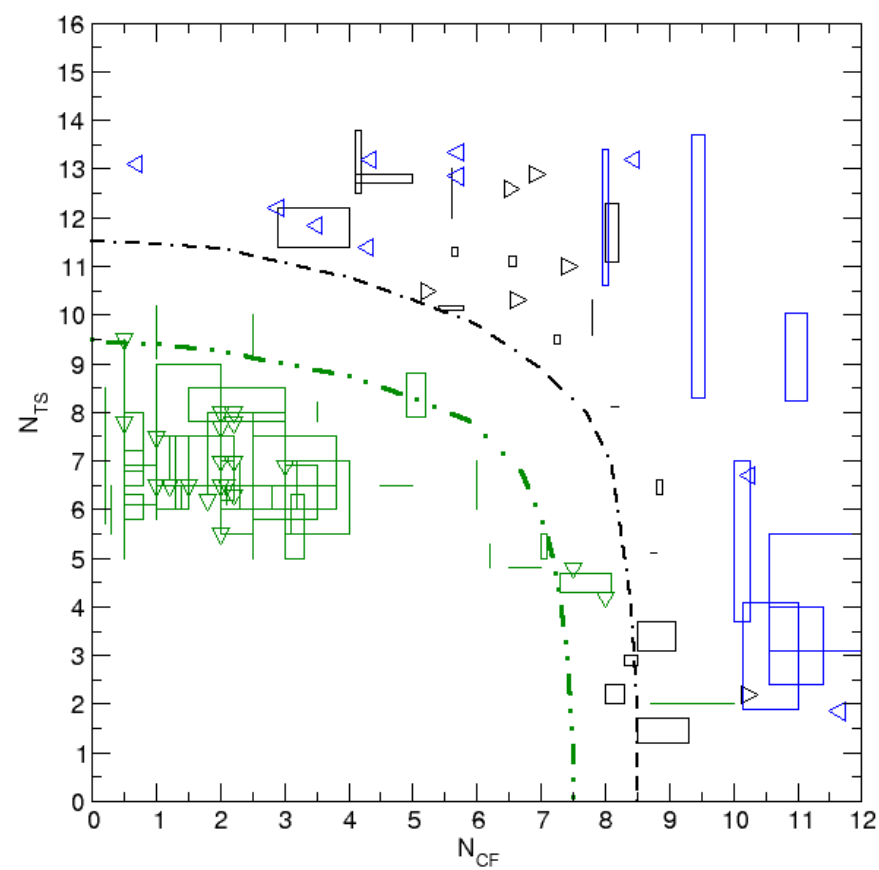

Fig. 20 Correlated transition $\left(N_{C F}, N_{T S}\right)$-factor pairs from Figure 15 in [14].

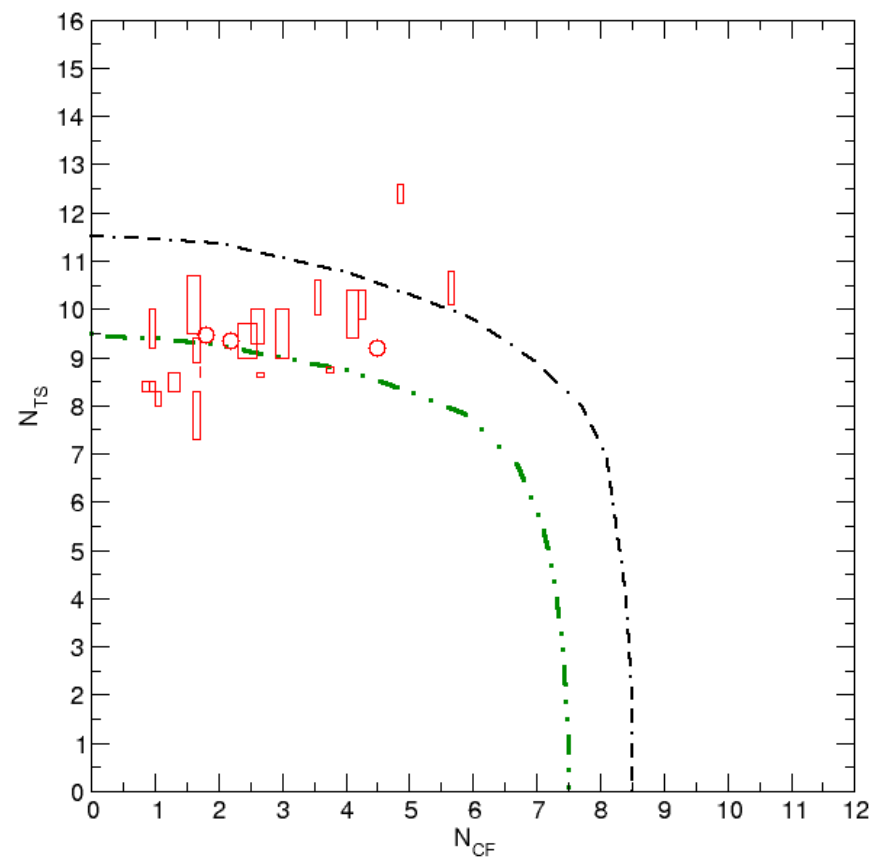

Fig. 21 Correlated transition $\left(N_{C F}, N_{T S}\right)$-factors of the cases from sections $\mathbf{V I}$ and VII. 


\section{Incompressible versus compressible stability theory}

Lately, a new discussion on the value for the transition $N_{T S}$-factor to be used with compressible stability theory has arisen in the context of the NASA NLF common research model and the Crossflow-Attenuated NLF design [17--20]. The question is whether $N_{T S}=10$ is adequate when using compressible stability theory.

To address this issue we re-evaluated all cases of sections $\mathrm{VI}$ and $\mathrm{VI}]$ with compressible stability theory. An example is shown in Figure 22 for a case with a flight altitude of $35000 \mathrm{ft}$, Mach number 0.78, and sideslip $0^{\circ}$. For this case, transition occurs at $36 \%$. With incompressible stability theory we obtain a transition $N_{T S}$-factor of 8.7 , whereas compressible theory gives the smaller value of $N_{T S}=5$ which is $40 \%$ smaller. To show that such a difference between incomressible and compressible $N_{T S}$-factors is typical for Mach 0.78 , we present in the Appendix the correlated $N_{T S}$-factors for the cases considered so far. For the cases of sections $\mathrm{VI}$ the average $N_{T S}$-factor obtained with incompressible theory is 9.2, whereas, with compressible theory, we have 5.4. A similar picture occurs for the cases of sections VII With incompressible theory we get an average transition $N_{T S}$-factor of 9.9, and with compressible theory 5.6.

Furthermore, we find that the difference between incompressible and compressible stability theory is smaller for the $N_{C F}$-factor. This is in line with earlier results [14].

We do not have any transition measurements from flight tests for Mach 0.85 . However, we project that the difference between incompressible and compressible $N_{T S}$-factors will become larger with increasing Mach number [21]. Therefore, we think that, if compressible stability theory is applied, a critical Tollmien-Schlichting $N$-factor of 10 is too large.

\section{Linear stability theory: some provisos}

Transition prediction with the $e^{N}$-method is semi-empirical. It consists of two different parts, a numerical part, consisting of boundary layer and stability calculations, and an empirical part, which is the $N$-factor correlation. Before we can apply the method, we have to calibrate it with transition experiments to determine a "transition $N$-factor." Having done this, we can apply the $e^{N}$-method for transition prediction in similar flows as follows: we calculate the $N$-factors with exactly the same numerical procedure and assume that transition occurs where the correlated transition $N$-factor is reached.

It is important to recognize that the application of linear stability calculations for transition prediction is not clear-cut. First, we can use different linear stability theories: local or non-local, incompressible or compressible, parallel or non-parallel, including surface curvature or not [22], as well as including wave front curvature or not [23]. In principle, though we can employ any combination of the above, certain combinations are regarded as best practice. Second, we can consider different instability modes in the boundary layer and trace their growth. We can compute the $N$-factor of a mode with a certain frequency and a specific second property, such as the propagation direction, the wave length, or the spanwise wave number. Thus, linear stability theory can be applied in many different ways. An overview of many different posible ways is given in [16], covering the evaluation work for the ELFIN Fokker F100 flight tests done by several organizations.

Linear stability theory (LST) provides a good approximation of the initial growth of an instability mode. However, transition is caused by nonlinear mode interaction and secondary as well as tertiary instabilities. With the $e^{N}$-method we are applying linear stability theory beyond its range of validity for small amplitudes, and extrapolating into the region of large amplitudes. Linear stability theory generates good results for boundary layers with a relatively long region with slowly growing modes, as occurs for NLF. and HLFC-wings. However, LST has to be applied with caution. 


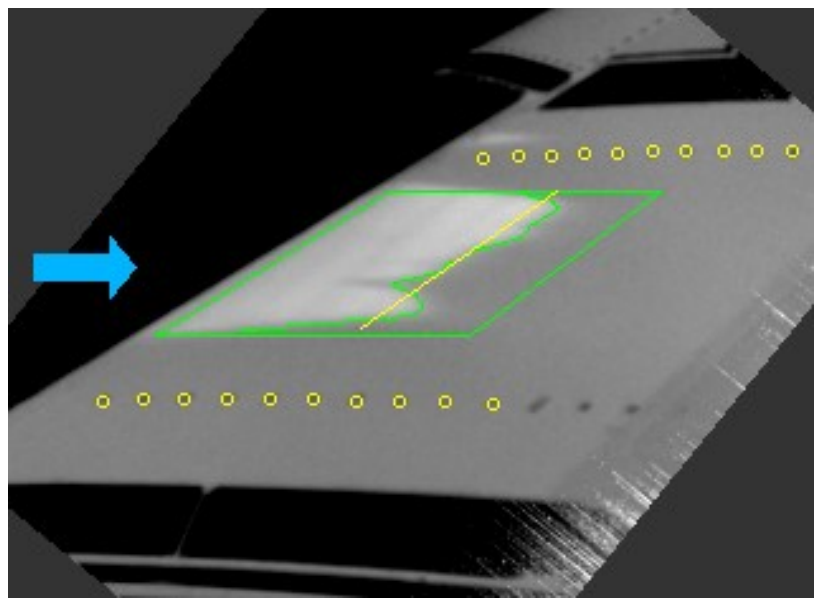

Fig. $22 h=35000 f t, M=0.78, \beta=0^{o}$, port side.

LILO-11:0410_1122 H 35 M 78 BETA 0.1 iH-1.0 S61 PORT NCOAMPESSIBLE WTHOUT CURVATURE

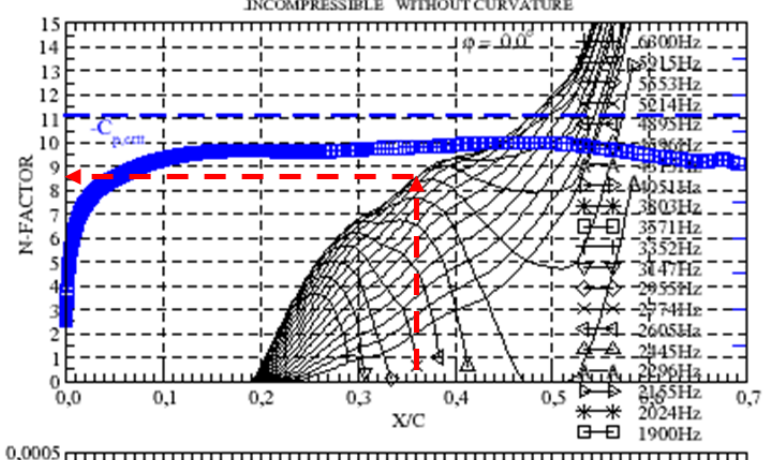

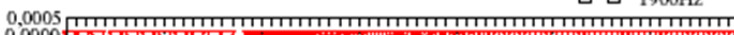
لَ,

(a) $N_{T S}$-factors obtained with incompressible theory.

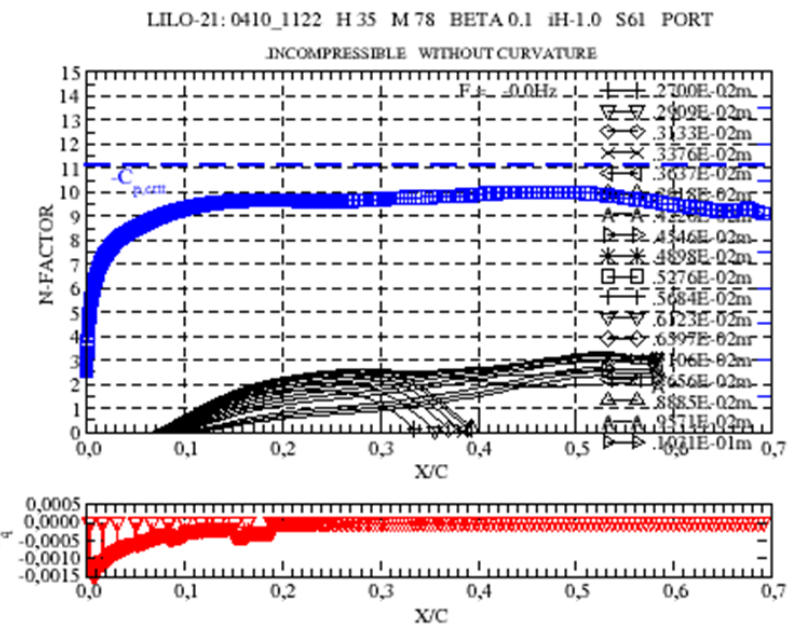

(c) $N_{C F}$-factors obtained with incompressible theory.

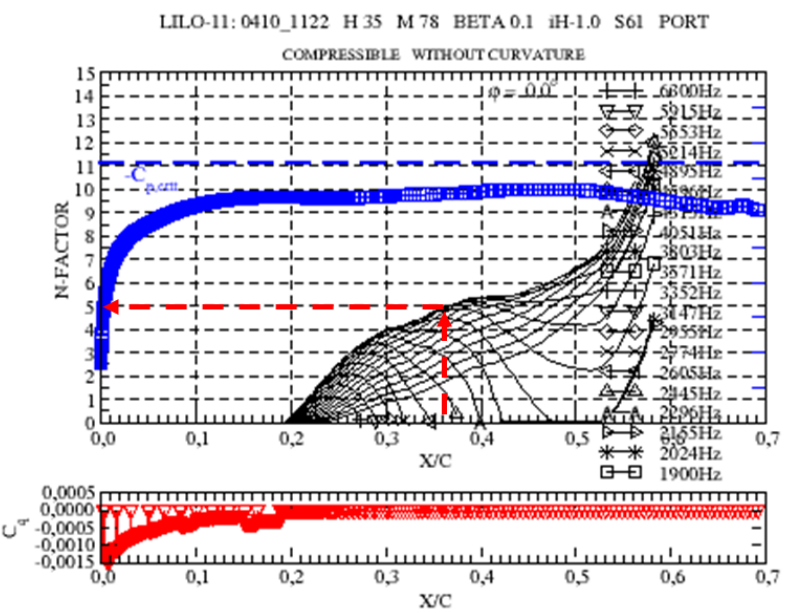

(b) $N_{T S}$-factors obtained with compressible theory.

LILO-21:0410 1122 H 35 M 78 BETA 0.1 iH-1.0 S61 PORT COAPRESSIBLE WTHOUTCURVATURE
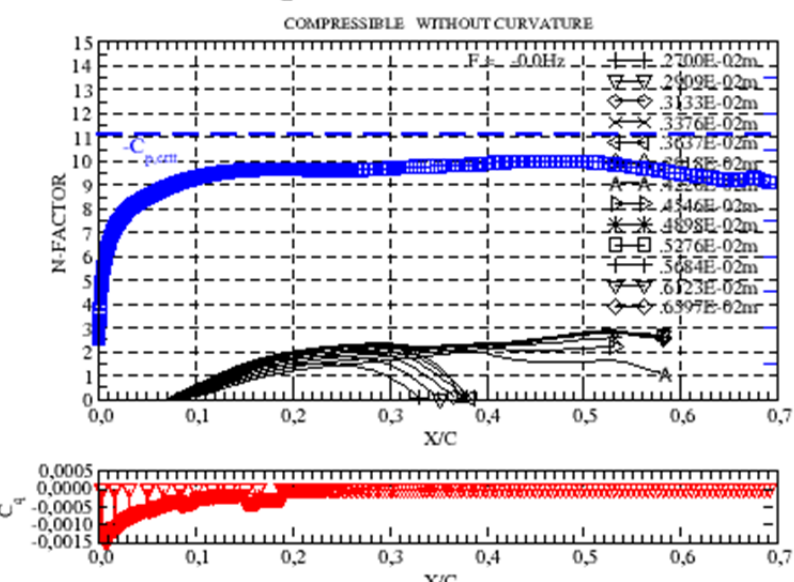

(d) $N_{C F}$-factors obtained with compressible theory.

Fig. $23 \quad N$-factors for the case of Figure 22 . 


\section{Appendix}

In Table 3 we present the correlated $N_{T C F}$ and $N_{T S}$-factors obtained with incompressible and compressible stability theory for the cases considered in section $\overline{\mathrm{VI}}$, including one additional case which is not in table 1 because the pressure distribution is not symmetric. All measurements were performed with flight altitude $35000 \mathrm{ft}$, Mach number 0.78 and sideslip angle $\beta \approx 0^{\circ}$. As usual, we consider a suitable spanwise region and determine for this region the earliest transition locations $X_{t r 1}$ as well as the latest one $X_{t r 2}$, and correlate $N$-factors. $N_{C F i 1}, N_{C F i 2}$ are the $N_{C F}$-factors, and $N_{T S i 1}, N_{T S i 2}$ the $N_{T S}$-factors obtained with incompressible stability theory, $N_{C F c 1}, N_{C F c 2}, N_{T S c 1}$, and $N_{T S c 2}$ are determined with compressible stability theory.

Table 3 Transition locations and correlated $N$-factors for $h=35000 \mathrm{ft}, M=0.78, \beta \approx 0^{o}$.

\begin{tabular}{|c|c|c|c|c|c|c|c|c|c|c|c|}
\hline Time & Side & $X_{t r 1}$ & $X_{t r 2}$ & $N_{C F i 1}$ & $N_{C F i 2}$ & $N_{T S i 1}$ & $N_{T S i 2}$ & $N_{C F c 1}$ & $N_{C F c 2}$ & $N_{T S c 1}$ & $N_{T S c 2}$ \\
\hline 103248 & port & $37 \%$ & $37 \%$ & 1.0 & 1.1 & 8.0 & 8.3 & 0.8 & 0.8 & 4.5 & 4.5 \\
103248 & starboard & $39 \%$ & $40 \%$ & 0.8 & 0.9 & 8.3 & 8.5 & 0.6 & 0.7 & 4.7 & 4.7 \\
103831 & port & $37 \%$ & $40 \%$ & 1.2 & 1.4 & 8.3 & 8.7 & 0.9 & 1.1 & 4.6 & 4.9 \\
103831 & starboard & $38 \%$ & $40 \%$ & 0.9 & 1.0 & 8.3 & 8.7 & 0.6 & 0.7 & 4.6 & 4.9 \\
105212 & port & $34 \%$ & $36 \%$ & 1.6 & 1.7 & 7.3 & 8.3 & 1.3 & 1.4 & 4.2 & 4.7 \\
105212 & starboard & $44 \%$ & $48 \%$ & 1.5 & 1.7 & 9.5 & 10.7 & 1.2 & 1.4 & 5.4 & 6.0 \\
111357 & starboard & $41 \%$ & $44 \%$ & 0.9 & 1.0 & 9.2 & 10.0 & 0.6 & 0.7 & 5.2 & 5.6 \\
111548 & starboard & $43 \%$ & $44 \%$ & 1.6 & 1.7 & 8.9 & 9.4 & 1.2 & 1.3 & 4.9 & 5.2 \\
111915 & port & $36 \%$ & $37 \%$ & 4.9 & 4.8 & 12.2 & 12.6 & 4.5 & 4.4 & 7.4 & 7.6 \\
111915 & starboard & $43 \%$ & $43 \%$ & 4.5 & 4.5 & 9.2 & 9.2 & 4.0 & 4.0 & 5.1 & 5.2 \\
112139 & port & $37 \%$ & $37 \%$ & 1.7 & 1.7 & 8.6 & 8.8 & 1.4 & 1.4 & 4.9 & 5.0 \\
112139 & starboard & $45 \%$ & $45 \%$ & 2.2 & 2.2 & 9.3 & 9.4 & 1.8 & 1.8 & 5.0 & 5.2 \\
112226 & port & $36 \%$ & $36 \%$ & 2.6 & 2.7 & 8.6 & 8.8 & 2.3 & 2.3 & 4.9 & 5.1 \\
112226 & starboard & $44 \%$ & $48 \%$ & 2.9 & 3.1 & 9.0 & 10.0 & 2.5 & 2.7 & 4.9 & 5.4 \\
112309 & port & $36 \%$ & $36 \%$ & 3.7 & 3.8 & 8.7 & 8.8 & 3.4 & 3.4 & 5.2 & 5.6 \\
112309 & starboard & $45 \%$ & $49 \%$ & 4.0 & 4.2 & 9.4 & 10.4 & 3.5 & 3.7 & 7.6 & 8.7 \\
\hline
\end{tabular}

Computing mean values, we obtain for the average transition $N_{T S}$-factors obtained with incompressible theory 9.2 and with compressible theory 5.4 .

In the following table we present the incompressible as well as the compressible $N$-factors for the cases of Table 2 The mean transition $N_{T S}$-factors, obtained with incompressible and compressible stability theory, are 9.9 and 5.6, respectively.

Table 4 Transition locations and correlated $N$-factors for $h=39000 \mathrm{ft}, M=0.78, \beta \approx 0^{\circ}$, starboard side.

\begin{tabular}{|c|c|c|c|c|c|c|c|c|c|c|c|}
\hline No. & Time & $X_{t r 1}$ & $X_{t r 2}$ & $N_{C F i 1}$ & $N_{C F i 2}$ & $N_{T S i 1}$ & $N_{T S i 2}$ & $N_{C F c 1}$ & $N_{C F c 2}$ & $N_{T S c 1}$ & $N_{T S c 2}$ \\
\hline 1 & 120513 & $48 \%$ & $48 \%$ & 1.8 & 1.8 & 9.4 & 9.5 & 1.5 & 1.5 & 5.1 & 5.2 \\
2 & 120621 & $47 \%$ & $49 \%$ & 2.3 & 2.6 & 9.0 & 9.7 & 2.1 & 2.3 & 5.0 & 5.4 \\
3 & 120700 & $47 \%$ & $50 \%$ & 2.5 & 2.7 & 9.3 & 10.0 & 2.1 & 2.3 & 5.2 & 5.8 \\
4 & 120730 & $50 \%$ & $51 \%$ & 3.5 & 3.6 & 9.9 & 10.6 & 3.0 & 3.1 & 5.6 & 5.9 \\
5 & 120803 & $49 \%$ & $50 \%$ & 4.2 & 4.3 & 9.8 & 10.4 & 3.7 & 3.8 & 5.5 & 5.9 \\
6 & 120833 & $49 \%$ & $51 \%$ & 5.6 & 5.7 & 10.1 & 10.8 & 5.1 & 5.2 & 5.7 & 6.3 \\
\hline
\end{tabular}




\section{Outlook and future work}

In this paper we present only some results from one flight performed within the AFLoNext project. However, several more flights are waiting for evaluation. We hope to be able to present further results in a follow-up paper.

\section{Acknowledgments}

The flight test activities received funding from the European Community's Seventh Framework Programme FP7/2007-2013, under grant agreement $n^{\circ}$ 604013, AFLoNext project. The activities were performed within AFLoNext Workpackage 1.1 with support of the partner Airbus Operations $\mathrm{GmbH}$, Airbus Operations S.A.S, Airbus Operations SL, Dassault Aviation SA, SONACA, TAI, AcQ Inducom, IBK Innovation, CIRA, DLR, FOI, Fraunhofer, and ONERA. Special thanks to Philipp Mühlmann (DLR, German Aerospace Center) for processing the IR-images, to Klaus de Groot (DLR, German Aerospace Center) for providing the flight test data, and to Nancy Schrauf for editing help.

\section{References}

[1] Schrauf, G., "Status and Perspective of Laminar Flow," The Aeronautical Journal, Vol. 109, No. 1102, 2005, pp. 639-644.

[2] Boeing Commercial Aircraft Group, "High Reynolds Number Hybrid Laminar Flow Control (HLFC) Flight Experiment II. Aerodynamic Design,” NASA CR-1999-209324, April 1999.

[3] Schrauf, G., and von Geyr, H., "Simplified Hybrid Laminar Flow Control for Transport Aircraft," In: Eberhardsteiner J. et al. (eds), European Congress on Computational Methods in Applied Sciences and Engineering (ECCOMAS 2012), 2012, Vienna, Austria.

[4] Braslow, A. L., "A History of Suction-Type Laminar-Flow Control with Emphasis in Flight Reserach," Monographs in Aerospace History, No.13, NASA History Division, 1999, Washington, DC.

[5] Horstmann, K. H., and Schröder, W., "A Simplified Suction System for a HLFC L/E Box of an A320 Fin,” ALTTA Technical Report, No. 23, 2001.

[6] Schrauf, G., and von Geyr, H., "Simplified Hybrid Laminar Flow Control for the A320 Fin. Aerodynamic and System Design. First Results," AIAA SciTech Forum, 6-10 January 2020, Orlando, Florida, USA, Paper 2020.1536.

[7] de Groot, K., "Quicklook: A C-Program to Check and Extract Pneumatic Pressures Recorded during the A320 HLFC Test Flights," DLR, 2018.

[8] Schrauf, G., "A Note on the Calculation of the Effective Sweep Angle and the Attachment Line Reynolds Number," ELFIN II Technical Report No. 116, January 1994.

[9] Schrauf, G., "LEA - A FORTRAN Subroutine to Compute the Effective Sweep Angle and the Attachment Line Reynolds Number." GSSC TR 10, 15.06.2012.

[10] "COCO - A Program to Compute Velocity and Temperature Profiles for Local and Nonlocal Stability Analysis of Compressible, Conical Boundary Layers with Suction,” ZARM Technik Report, November 1998.

[11] Schrauf, G., "LILO 2.1 User's Guide and Tutorial," Bremen, Germany, GSSC Technical Report No. 6, originally issued 2004, modified for Version 2.1 July 2006.

[12] Akima, H., "A New Method of Interpolation and Smooth Curve Fitting Based on Local Procedures." Journal of the Association for Computing Machinery, Vol. 17,No. 4, Oct. 1970, pp. 589-602.

[13] Henke, R., “The Airbus A320 HLF Fin Programme,” Nouvelle Revue d'Aéronautique et d'Astronautique, No.2, $1998,53-55$.

[14] Schrauf, G, "Large-Scale Laminar-Flow Tests Evaluated with Linear Stability Theory,” AIAA Journal of Aircraft, Vol. 41, No. 2, March-April 2004, pp. 224 - 230.

[15] Schrauf, G, "Transition Prediction for the Design of Laminar Surfaces," Lecture Series at the University of São Paulo, São Carlos, Brazil , August 2011.

[16] Schrauf, G., Perraud, J., Vitiello, F., Lam, F., Stock, H.W., Abbas, A., "Transition Prediction with linear stability theory. Lessons learned from the ELFIN F100 flight demonstrator," 2nd European Forum on Laminar Flow Technology, 10.-12. 6. 1996, Bordeaux. Proceedings published by AAAF. 6, Rue Galilée, 75782 Paris, France. 
[17] Campbell, R.L., Lynde, M.N., "Building a Practical Natural Laminar Flow Design Capability," 35th AIAA Applied Aerodynamics Conference Denver, 5-9 June 2017, Denver, Colorado, AIAA 2017-3059.

[18] Lynde, M.N., Campbell, R.L., "Computational Design and Analysis of a Transonic Natural Laminar Flow Wing for a Wind Tunnel Model," 35th AIAA Applied Aerodynamics Conference Denver, 5-9 June 2017, Denver, Colorado, AIAA $2017-3058$.

[19] Lynde, M.N., Campbell, R.L., Rivers, M.B., Viken, S.A., Chan, D.T., Watkins, A.N., Goodliff, S.L., "Preliminary Results from an Experimental Assessment of a Natural Laminar Flow Design Method," AIAA SciTech Forum, 7-11 January 2019, San Diego, California, AIAA 2019-2298.

[20] Streit, T., Seitz, A., Kunze, P., Hein, S., "NLF Potential of Laminar Transonic Long Range Aircraft," Accepted for AIAA Aviation Forum 2020.

[21] Atkin, C., Schrauf, G., "Progress in linear stability methods for design applications.” In: ECCOMAS 2000, 11-14 September 2000, Barcelona, Spain, CD-ROM Proceedings by ECCOMAS, ISBN: 84-89925-70-4.

[22] Schrauf, G., "Curvature effects for three-dimensional compressible boundary-layer stability," Z. Flugwiss. Weltraumforsch. 16 (1992), 119-127.

[23] Schrauf, G., “On Wave-Front Curvature in Linear Stability Theory,” Rech. Aérosp., 1994, No. 2, pp. 153-156.

[24] Schrauf, G., Herbert, Th., Stuckert, G., "Evaluation of Transition in Flight Tests Using Nonlinear Parabolized Stability Analysis." AIAA JoA Vol. 33, No. 3, May-June 1996, pages 554-560. 